\title{
FOUR-DIMENSIONAL HETEROTIC STRINGS-ORBIFOLDS AND COVARIANT LATTICES
}

\author{
Dieter LÜST and Stefan THEISEN \\ Max-Planck-Institut für Physik und Astrophysik, Werner-Heisenberg-Institut für Physik, \\ P.O. Box 4012 12, Munich, Fed. Rep. Germany
}

Received 17 November 1987

\begin{abstract}
Two - so far unrelated - constructions of four-dimensional heterotic string theories are discussed within a common framework. We show that four-dimensional heterotic string theories which are based on covariant self-dual lattices are equivalent to a wide class of asymmetric orbifolds. This equivalence provides an explicit realization of twist fields and allows the straightforward calculation of scattering amplitudes of various massless fields. "Topological" properties of the orbifolds, like the number of fixed points, are related to group theoretical features of the covariant lattices. Two explicit examples illustrate our conclusions.
\end{abstract}

\section{Introduction}

One of the major fields of interest in string theory [1] is the construction of four-dimensional string theories [2-11]. The development started with the CalabiYau compactification [12] of 10-dimensional heterotic strings [13]. At the same time, it was also recognized [14] that conformal invariance puts severe constraints to the possible background geometry the string is propagating in. Thus the problem of finding four-dimensional string theories is reduced to classifying all two dimensional (super) conformal field theories (obeying certain consistency conditions) where four variables play the role of the space-time coordinates.

One background which certainly satisfies the constraints of conformal invariance is the compactification of 10-dimensional string theories on simple tori $[2,15]$. However, torus compactification leads to nonchiral, $N=4$ supersymmetric theories in 4 dimensions which are phenomenologically uninteresting. In order to circumvent these difficulties orbifold compactifications $[6,7,16-20]$ were discussed. These are obtained by modding out of the symmetry group of the torus the string is compactified on an automorphism group in such a way that the compact space is singular at the fixed points of this symmetry.

Finally, four-dimensional chiral string theories [3-5] were constructed recently without reference to any geometrical compactification. Fermion-boson equivalence in 2-dimensional conformal field theory leads to equivalent fermionic respectively 
bosonic description of these theories. In the fermionic construction $[3,5]$ all internal degrees of freedom are regarded as free fermions, whereas the latter method - the covariant lattice approach $[4,10,21-25]$ - is based on the bosonization of the Ramond-Neveu-Schwarz (RNS) $[26,27]$ fermionic string. In both cases left and right movers are treated as totally independent degrees of freedom. Therefore, these theories can be regarded in a way as asymmetric orbifolds [7]. The objective of the present paper is to make this relationship clearer. This also allows us to retain some of the geometrical interpretations of these models.

Specifically, we will consider the case where the (asymmetric) orbifold is obtained by modding out an inner automorphism of the lattice of the left (right) moving momentum vectors obtained from the torus compactification. In this case the modding can be represented as a shift on the lattice. Taking also the bosonized NSR fermions and superconformal ghosts into account, the lattice shift maps an odd self-dual lattice again onto an odd self-dual lattice. (This was already remarked in [7].) The shift or equivalently Lorentz rotation in the lorentzian self-dual lattice can reduce the number of supersymmetries and create a chiral fermion spectrum. However, the rank (22) of the gauge group is not reduced.

Since the equivalence between modding out a rotational symmetry and shifting the lattice is true only if the inner automorphism of the "spatial" lattice the heterotic string is compactified on is realized on the momentum lattice, a very special choice of background parameters of the torus compactification is needed. It means that the covariant self-dual lattices correspond to singular points in the parameter space of all consistent (asymmetric) orbifolds.

These points are characterized by a dramatic enhancement of the (gauge and global) symmetries of the theory. More generally, since the orbifolds are regarded as limiting cases of more general consistent string backgrounds (like Calabi-Yau manifolds) the theories based on the self-dual lattices are the highest degenerate cases.

Even if (probably) not leading to phenomenologically correct theories, the covariant lattices are very useful to study interactions of the massless fields in the theory [23]. Here, all vertex operators are explicitly given in terms of bosonic free fields. Since the massless particle spectrum is completely described by the conformal properties of the vertex operators (plus the transformations under model dependent symmetries), the scattering amplitudes obtained in the covariant lattice approach remain also valid in a more general background resp. conformal field theory. The number of chiral fermion families, a topological property of the compact manifold, reflects itself in symmetries of the covariant lattices. Transition rules for the Yukawa couplings, related to topological intersection matrices, are given by Clebsch-Gordan coefficients of the symmetry group of the covariant lattice.

The paper is organized as follows: In sect. 2 we recall basic properties of torus compactification of heterotic strings and the construction of orbifolds. We especially discuss the case where the modding by an inner automorphism can be realized 
also as a lattice shift. In sect. 3 we prove that these types of asymmetric orbifolds are equivalently described by (covariant) self-dual lattices. We also relate some of the (topological) properties of the orbifolds to the self-dual lattices and explicitly construct the twist field vertex operators of the orbifolds. This is illustrated in sect. 4 by two explicit examples - the well known $\mathrm{Z}$-orbifold and a $\mathrm{Z}_{4}$-orbifold leading to a $D_{n}$ lattice already discussed in refs. $[4,23]$. Sect. 5 summarizes the paper.

\section{Torus compactification and $\mathbf{Z}_{N}$-orbifolds}

Let us start by recalling the compactification of the 10-dimensional, untwisted, supersymmetric heterotic string theory [13]. In 10 dimensions 16 left moving coordinates are "compactified" on a torus with fixed radii such that the left moving momenta $p_{L}^{I}(I=1 \ldots 16)$ span an even self-dual lattice. In 16 dimensions there are just two of them, the root lattice of $E_{8} \times E_{8}$ and the weight lattice of Spin (32) $/ Z_{2}$. On the other hand, the right moving sector corresponds to the superstring which leads to a $N=1$ supergravity theory in 10 dimensions. Both, the appearance of the two gauge groups as well as the space-time super-symmetry are enforced by modular invariance of the left respectively right moving sector.

The most obvious way to get a 4-dimensional string model is to compactify the extra six dimensions again on a torus.

The 6-dimensional torus $\mathrm{T}^{6}=\mathrm{R}^{6} / 2 \pi \Lambda_{6}$ is generated by modding out of 6 space dimensions a lattice $\Lambda_{6}$, i.e. identifying points $X^{\mu}$ and $X^{\mu}+2 \pi n^{i}\left(e_{i}\right)^{\mu}(\mu, i=1 \ldots 6)$ where the $e_{i}$ are a set of basic vectors for the six-dimensional lattice $\Lambda_{6}$. The winding vectors are given by $L^{\mu}=n^{i} e_{i}^{\mu}, L \in \Lambda_{6}$, and indicate how many times the closed string is wrapped around the torus.

Modular invariance yields that the quantized momenta $\tilde{p}_{\mathrm{L}}^{i}, \tilde{p}_{\mathrm{R}}^{i}$ together with the momenta $p_{L}^{I}$ must span an even, self-dual lorentzian lattice $\Gamma_{22 ; 6}[2]^{\star}$ where the signature of this lattice is defined as $\boldsymbol{p}^{2}=-\boldsymbol{p}_{\mathrm{L}}^{2}-\tilde{\boldsymbol{p}}_{\mathrm{L}}^{2}+\tilde{\boldsymbol{p}}_{\mathrm{R}}^{2}, \boldsymbol{p}=\left(\boldsymbol{p}_{\mathrm{L}}, \tilde{\boldsymbol{p}}_{\mathrm{L}}, \tilde{\boldsymbol{p}}_{\mathrm{R}}\right)$.

An important feature of all even self-dual lorentzian lattices $\Gamma_{22 ; 6}$ is that they are isomorphic to each other, i.e. they can be obtained from each other by $\operatorname{SO}(22,6)$ transformations. Given the mass formula of the toroidally compactified heterotic string,

$$
\begin{aligned}
\frac{1}{8} m_{\mathrm{L}}^{2} & =\frac{1}{2}\left(\boldsymbol{p}_{\mathrm{L}}^{2}+\tilde{\boldsymbol{p}}_{\mathrm{L}}^{2}\right)+N_{\mathrm{L}}-1, \\
\frac{1}{8} m_{\mathrm{R}}^{2} & =\frac{1}{2} \tilde{\boldsymbol{p}}_{\mathrm{R}}^{2}+N_{\mathrm{R}}-1, \\
m^{2} & =\frac{1}{2}\left(m_{\mathrm{L}}^{2}+m_{\mathrm{R}}^{2}\right), \quad m_{\mathrm{L}}^{2}=m_{\mathrm{R}}^{2},
\end{aligned}
$$

it is evident that the spectrum is only invariant under the maximal compact

${ }^{\star}$ Note that we denote by $\Lambda$ the lattice which defines the torus the coordinates are compactified on, whereas $\Gamma$ always corresponds to the generalized momentum lattice. For arbitrary background fields they are not necessarily dual to each other. 
subgroup $\mathrm{SO}(6) \times \mathrm{SO}(22) \subset \mathrm{SO}(22,6)$. Therefore, different compactifications are obtained by varying $\operatorname{dim}(\mathrm{SO}(22,6) / \mathrm{SO}(22) \times \mathrm{SO}(6))=132$ continuous parameters. These can be interpreted [15] as background fields on the torus, namely constant metric fields $g_{i j}=e_{i} \cdot e_{j}$, determining the radii and angles of the underlying torus, constant antisymmetric tensor fields $B_{i j}$ and constant Wilson lines $A_{i}^{l}$. In the presence of these background fields the quantized momenta $\boldsymbol{p}_{\mathrm{L}}, \tilde{\boldsymbol{p}}_{\mathrm{L}}, \tilde{\boldsymbol{p}}_{\mathrm{R}}$ (with $\boldsymbol{p}_{\mathrm{L}}=p_{\mathrm{L}}^{I} e_{I}$ etc.) take the following form:

$$
\begin{aligned}
& p_{\mathrm{L}}^{I}=g^{I J} m_{J}+A_{i}^{I} n^{i}, \\
& \tilde{p}_{\mathrm{L}}^{i}=n^{i}+\frac{1}{2} g^{i j} m_{j}-g^{i j} B_{j k} n^{k}-\frac{1}{2} g^{i j} A_{j}^{I}\left(m_{I}+\frac{1}{2} g_{I J} n^{k} A_{k}^{J}\right), \\
& \tilde{p}_{\mathrm{R}}^{i}=-n^{i}+\frac{1}{2} g^{i j} m_{j}-g^{i j} B_{j k} n^{k}-\frac{1}{2} g^{i j} A_{j}^{I}\left(m_{I}+\frac{1}{2} g_{I J} n^{k} A_{k}^{J}\right) .
\end{aligned}
$$

The $g^{I J}$ 's are the inverse metric of the self-dual lattices $\mathrm{E}_{8} \otimes \mathrm{E}_{8}$ and $\operatorname{Spin}(32) / \mathrm{Z}_{2}$ respectively. (Thus we have that $\Gamma_{16}=\Lambda_{16}$ for $A_{i}^{I}=0$.)

All toroidal compactifications lead to 4-dimensional heterotic string theories with the rank 22 gauge group $(\mathrm{U}(1))^{22}$ with gauge fields corresponding to the internal oscillator excitations. This gauge symmetry can be enlarged by the Frenkel-Kac mechanism [28] for very special values of the background fields $g_{i j}, B_{i j}, A_{i}^{I}$. Consider [29] e.g. a compactification of the $\mathrm{E}_{8} \times \mathrm{E}_{8}$ heterotic string theory with winding vectors being elements of the root (weight) lattice $\Lambda_{6}$ of a simply laced rank 6 gauge group $\mathrm{G}$. This fixes some of the radii and angles of the 6-dimensional torus.

For arbitrary $B_{i j}$ and $A_{i}^{I}=0$ one has the gauge group $\mathrm{E}_{8} \times \mathrm{E}_{8} \times[\mathrm{U}(1)]^{6}$ after compactification. However, for a very particular background $B_{i j}$ the gauge symmetry is enlarged to $\mathrm{E}_{8} \times \mathrm{E}_{8} \times \mathrm{G}$. In this case, the $B_{i j}$ 's are chosen such that $\tilde{\boldsymbol{p}}_{\mathrm{L}} \in \operatorname{root}$ vectors of $G$ lead to additional massless vector particles. This means that the symmetry of the spatial lattice $\Lambda_{6}$ is also realized on the six-dimensional part $\Gamma_{6 ; 6}$ of the momentum lattice $\Gamma_{22 ; 6}$, i.e. $\Gamma_{6 ; 6}$ is the root (weight) lattice of $\mathrm{G}_{\mathrm{L}} \times \mathrm{G}_{\mathrm{R}}$. Similarly, special values for $A_{i}^{I}$ can enhance the $\mathrm{SO}(32) \times(\mathrm{U}(1))^{6}$ gauge symmetry to $\mathrm{SO}(44)$. In both examples any small modification of the background parameters gives mass to the charged vector bosons. Furthermore, not only massless vector particles plus their supersymmetric partners exist for a particular background but also massless scalars and fermions. They become massive for arbitrary background and build the longitudinal components of the massive vector multiplets realizing a kind of (super) Higgs effect.

The reason that these models allow for continuous Lorentz rotations of the self-dual lattice $\Gamma_{22 ; 6}$ is that the rotations do not affect the 2-dimensional right-moving world-sheet supercurrent $T_{\mathrm{F}}$. It is given by:

$$
T_{\mathrm{F}}=-\frac{1}{2}\left(\sum_{\mu=1}^{4} \partial X_{\mu} \psi^{\mu}+\sum_{i=1}^{6} \partial X_{i} \psi^{i}\right) .
$$


Superconformal invariance is maintained trivially, since the boundary conditions of the two-dimensional RNS fermions $\psi^{\mu}, \psi^{i}$ are not changed by the torus compactification (the $\partial X_{i}$ are also still periodic!). In fact, the torus compactification does not act on the fermionic coordinates of the right moving superstring at all. Thus, the 10-dimensional gravitinos remain massless in four dimensions leading to a $N=4$ supergravity theory. The resulting matter fermions are necessarily non-chiral and the continuous Lorentz rotations do not change the chiral structure of the theory.

In order to get a chiral theory and simultaneously also to get rid of at least 3 gravitinos one has to divide the theory by symmetries under which chiral partners and unwanted gravitinos are not invariant. Specifically we consider the following asymmetric orbifold construction:

$$
\mathrm{O}_{22 ; 6}=\frac{\mathrm{T}_{\mathrm{L}}^{16}}{g_{\mathrm{L}}} \otimes \frac{\mathrm{T}_{\mathrm{L}}^{6}}{\tilde{g}_{\mathrm{L}}} \otimes \frac{\mathrm{T}_{\mathrm{R}}^{6}}{\tilde{g}_{\mathrm{R}}} .
$$

$g_{\mathrm{L}}, \tilde{g}_{\mathrm{L}}, \tilde{g}_{\mathrm{R}}$ are the discrete $\mathrm{Z}_{N}$ symmetries of the left- resp. right-moving tori the coordinates $X_{\mathrm{L}(\mathrm{R})}$ live on. In particular we will consider the case where $g=g_{\mathrm{L}} \times \tilde{g}_{\mathrm{L}}$ $\times \tilde{g}_{R}$ is a discrete subgroup of a simply laced group $G$ of which the lattice $\Lambda$ is the root resp. weight lattice.

We call $\mathrm{O}_{22 ; 6}$ a symmetric orbifold if $\tilde{g}_{\mathrm{L}}=\tilde{g}_{\mathrm{R}}$. Only in this case one can regard the models as compactified string theories.

In this paper we want to consider two types of $Z_{N}$ moddings, namely dividing out by a translational or by a rotational discrete symmetry of the torus. We can get a translational discrete symmetry of order $N$ by using a vector $\delta(\mathrm{N} \delta \in \Lambda)$ as follows:

$$
g(X)=X \pm 2 \pi \delta .
$$

In the mode expansion of the shifted string the winding vectors $L$ are replaced by $\boldsymbol{L}+\boldsymbol{\delta}$. For the rotational $Z_{N}$ symmetries one has to ensure that the space-time lattice $\Lambda$ has these symmetries. We consider the case of compactification on weight lattices of simply laced groups. The action of $g$ on the compactified coordinates (in a diagonal basis) reads:

$$
g\left(X_{i}\right)=\mathrm{e}^{2 \pi i \eta_{i}} X_{i}
$$

For the twisted strings the boundary conditions $X(\sigma+\pi)=g(X(\sigma)) \bmod \Lambda$ do not allow for quantized momenta or winding states and the mode expansion contains oscillators $\alpha_{m+1 / N}^{i}$. These lead to states with fractional oscillator number, and the center of mass positions are quantized and correspond to fixed points of the discrete symmetry group $g$.

A particularly interesting case is obtained if the background parameters of the underlying torus have special values such that the symmetry $g$ of the space-time 
lattice $\Lambda$ is realized also on the generalized momentum lattice $\Gamma_{22 ; 6}$ i.e. $\Gamma_{22 ; 6}$ is again a root (weight) lattice of $\mathrm{G}$. Then, the shifts resp. rotations are realized directly in $\Gamma_{22 ; 6}$ a fact which makes the discussion of modular invariance and superconformal invariance more transparent.

For the translational case an arbitrary, now asymmetric element $\hat{g}$ acts on the winding degrees of freedom of the Hilbert space as:

$$
\hat{g}|\boldsymbol{p}\rangle=\hat{g}\left|\boldsymbol{p}_{\mathrm{L}}, \boldsymbol{p}_{\mathrm{R}}\right\rangle=|\boldsymbol{p}+\boldsymbol{\delta}\rangle=\left|\boldsymbol{p}_{\mathrm{L}}+\boldsymbol{\delta}_{\mathrm{L}}, \boldsymbol{p}_{\mathrm{R}}+\boldsymbol{\delta}_{\mathrm{R}}\right\rangle \text {. }
$$

Therefore, we express $\hat{g}$ by the generators of the Cartan subalgebra of $\mathrm{G}$ :

$$
\hat{g}=\prod_{i} \exp \left(2 \pi i \delta_{i} H^{i}\right), \quad i=1, \ldots, \operatorname{rank} \mathrm{G} .
$$

This element acts on the right moving RNS fermionic coordinates as follows:

$$
\hat{g}_{\mathrm{R}}\left(\psi_{i}\right)=\mathrm{e}^{2 \pi i \delta_{i \mathrm{R}}^{\prime}} \psi_{i}
$$

Modular invariance demands that the full Hilbert space is obtained by summing over all shifted sectors of the theory. In the unshifted sector one has to consider only $g$-invariant states $\mid p_{0}>\left(p_{0} \in \Gamma_{22 ; 6}\right)$ which satisfy

$$
\boldsymbol{p}_{\mathrm{OL}} \cdot \boldsymbol{\delta}_{\mathrm{L}}-\boldsymbol{p}_{0 \mathrm{R}} \cdot \boldsymbol{\delta}_{\mathrm{R}}=N_{\boldsymbol{\delta}_{\mathrm{R}}^{\prime}}
$$

where $N_{\delta_{\mathrm{R}}^{\prime}}$ denotes the eigenvalue of the right moving RNS states under the considered shift. Therefore, the invariant states are defined by a combined GSOprojection in the bosonic and fermionic part of the theory which implies a non-trivial interplay between the left- and right-moving coordinates in a chiral theory.

The Hilbert space in the shifted sector is built by states $\left|\boldsymbol{p}_{0}+\boldsymbol{\delta}\right\rangle, \mid \boldsymbol{p}_{0}+$ $2 \delta\rangle, \ldots,\left|p_{0}+(N-1) \delta\right\rangle$. Thus, the Hilbert space is characterized by shifted "lattices" $\Gamma_{0}, \Gamma_{0}+\delta, \Gamma_{0}+2 \delta, \ldots$ These are of course no longer self-dual lattices. In the next chapter we will explain how to complete the shifted lattices by the lattice of the bosonized (twisted) fermionic coordinates to obtain again a self-dual lattice.

The states in the shifted sector of the theory are simply created by soliton type vertex operators $\exp i\left(\boldsymbol{p}_{0}+\boldsymbol{\delta}\right) \cdot \boldsymbol{X}$ where $\boldsymbol{X}$ are the compactified coordinates. The non-trivial shift gives an extra contribution to the Virasoro operators $L_{0_{\mathrm{L}}}, L_{0_{\mathrm{R}}}$ such that the change of the ground state energies is given by:

$$
\begin{aligned}
& \Delta L_{0 \mathrm{~L}}=\frac{1}{2} \boldsymbol{\delta}_{\mathrm{L}}^{2}, \\
& \Delta L_{0 \mathrm{R}}=\frac{1}{2} \boldsymbol{\delta}_{\mathrm{R}}^{2}+\frac{1}{2} \boldsymbol{\delta}_{\mathrm{R}}^{\prime 2} .
\end{aligned}
$$

Modular invariance implies the level matching condition

$$
\Delta L_{0 \mathrm{~L}}=\Delta L_{0 \mathrm{R}} \bmod 1 \text {. }
$$


Now, since $g$ acts trivially on the oscillator (Cartan subalgebra) degrees of freedom, the rank of the gauge group can never be reduced by modding out a translational symmetry from the lattice $\Gamma_{22 ; 6}$.

Let us now discuss the modding of a rotational discrete symmetry. A rotation in $\Gamma_{22 ; 6}$ can be represented by the following element $\hat{g}$ :

$$
\hat{g}=\prod_{i} \exp \left(2 \pi i \eta_{i} J_{i}\right)
$$

The $J_{i}$ are the non-commuting generators of G. Specifically, we want to consider Weyl rotations in $\Gamma_{22 ; 6}$ i.e. inner automorphisms of the lattice $\Gamma_{22 ; 6}$. Then $\hat{g}$ can be represented in $\Gamma_{22 ; 6}$ as follows:

$$
\hat{g}_{\{\boldsymbol{\alpha}\}}=\prod_{\{\boldsymbol{\alpha}\}} \exp \left[i \pi\left(E_{\boldsymbol{\alpha}}+E_{-\boldsymbol{\alpha}}\right)\right]=\prod_{\{\boldsymbol{\alpha}\}} \omega_{\boldsymbol{\alpha}} .
$$

$E_{\alpha}$ are the non-commuting generators of $\mathrm{G}$ in the Cartan-Weyl basis where $\alpha$ is the corresponding (length) ${ }^{2}=2$ root vector in $\Gamma_{22 ; 6}$. The single element $\omega_{\alpha}$ represents a reflection of a lattice vector $\boldsymbol{p}$ on the hyperplane orthogonal to the root $\alpha$ on the root lattice:

$$
\omega_{\alpha} p \omega_{\alpha}^{-1}=p-\alpha(\alpha \cdot p) .
$$

Thus $\hat{g}$ acts on a momentum state $|\boldsymbol{p}\rangle$ like:

$$
\hat{g}|\boldsymbol{p}\rangle=|\hat{g}(\boldsymbol{p})\rangle:=\left|\hat{g} \boldsymbol{p} \hat{g}^{-1}\right\rangle .
$$

To construct the Hilbert space in the untwisted sector one has again to project onto states invariant under the rotation. Here the oscillator excitations are in general not invariant under the rotational symmetry. Therefore, one expects that the rank of the gauge symmetry is reduced by the modding. For the special choice of background parameters we are considering, this is however not true. "New" U(1) gauge bosons are obtained by invariant combinations of root vectors:

$$
\begin{aligned}
\mid \mathrm{U}(1) \text { gauge boson }\rangle & \left.=|\boldsymbol{\alpha}\rangle+|\hat{g} \boldsymbol{\alpha}\rangle+\left|\hat{g}^{2} \boldsymbol{\alpha}\right\rangle+\cdots+\hat{g}^{N-1} \boldsymbol{\alpha}\right\rangle \\
& =\sum_{i=0}^{N-1}\left|\boldsymbol{\alpha}_{i}\right\rangle, \quad \sum_{i=0}^{N-1} \boldsymbol{\alpha}_{i}=\mathbf{0} .
\end{aligned}
$$

This is true only if we divide the rotational symmetry out of the momentum lattice $\Gamma_{22 ; 6}$. For arbitrary backgrounds no massless states correspond to the invariant root combinations, and the rank of the gauge group will be lowered.

Modular invariance enforces to take into account also the twisted sector. Here, no winding states with $\boldsymbol{p}_{\mathrm{L}}, \boldsymbol{p}_{\mathrm{R}} \neq \mathbf{0}$ are allowed. States are just characterized by non- 
integer oscillators obeying the following boundary conditions:

$$
\hat{g}\left(\partial X_{i}\right)=\exp \left(2 \pi i \eta_{i}\right) \partial X_{i}
$$

Furthermore, the states in the twisted sector are degenerate where the degeneracy factor in the left (right) moving sector is given by

$$
D_{\mathrm{L}(\mathrm{R})}=\sqrt{\frac{\operatorname{det}\left(1-\hat{g}_{\mathrm{L}(\mathrm{R})}\right)}{\left|\Gamma_{0}^{*} / \Gamma_{0}\right|}} .
$$

For a symmetric orbifold this factor becomes

$$
D_{\mathrm{L}+\mathrm{R}}=\operatorname{det}(1-\hat{g})=N_{\mathrm{F}} \text {. }
$$

$N_{\mathrm{F}}$ is the number of fixed points of $g$ indicating that the center-of-mass coordinates are located at the fixed points of the symmetry group. Compactifying the heterotic string on the weight lattice $\Lambda_{6 ; 6}$ of $\mathrm{G}_{\mathrm{L}} \times \mathrm{G}_{\mathrm{R}}\left(\mathrm{G}_{\mathrm{L}}=\mathrm{G}_{\mathrm{R}}=\mathrm{G}\right), N_{\mathrm{F}}$ is given by

$$
N_{\mathrm{F}}=\text { order of center of } \mathrm{G} \text {. }
$$

Physical states in the twisted sector are generated, besides the fractional oscillators, by twist fields $\sigma[30,31]$ which introduce branch cuts into the operator algebra,

$$
\partial_{z} X(z) \sigma(w)=(z-w)^{-(1-(\eta / N))} \tau(w)
$$

where $\tau(w)$ is an excited twisted field. The twist fields have definite conformal dimensions such that the change in zero point energy,

$$
\Delta L_{0}=\frac{1}{4} \sum_{i} \eta_{i}\left(1-\eta_{i}\right)
$$

is exactly compensated by these fields. However, the twist fields are not known explicitly and cannot be expressed in terms of free bosonic fields. This fact makes the calculation of scattering amplitudes involving twist fields rather complicated and involves the solution of a system of differential equations [32] to obtain the correlation functions of these fields.

Since the right-moving world-sheet fermions $\psi^{i}(z)$ and the compactified bosonic oscillators $\partial X_{i}(i=1 \ldots 6)$ have twisted boundary conditions one has to discuss the preservation of the world sheet supersymmetry. The right-moving world-sheet supercurrent $T_{F}$ has the same form (eq. (2.3)) as for the torus compactification. World sheet supersymmetry demands that $T_{\mathrm{F}}$ has definite boundary conditions. Since $\psi^{\mu}$ is either periodic - the Ramond sector - leading to space-time fermions or antiperiodic - the Neveu-Schwarz sector - with space-time bosons, and $\partial X_{\mu}$ is 
periodic under the twist, $\partial X_{i} \psi^{i}$ must have also periodic resp. antiperiodic boundary conditions. This requirement restricts the possible shifts or rotations in the right part of $\Gamma_{22 ; 6}$. This problem was discussed in ref. [6] with the result that for a special model rotations which are in the Weyl group of $G_{R}$ preserve world sheet supersymmetry. This seems necessary for a general asymmetric orbifold, however not for a symmetric orbifold. Thus we restrict ourselves to consider these types of rotational symmetries as mentioned before.

So far we have described the modding by a rotational resp. translational symmetry as two independent cases leading to different kinds of orbifold theories in four dimensions. This is, however, not true. The automorphisms which are in the Weyl group of $G$ and the shifts are in fact equivalent. (See also refs. [7, 16] and references therein.) The equivalence has not to be understood to mean that the compactified coordinates are in one to one correspondence, but the spectra and partition functions are identical for the Weyl rotation resp. shifting. This implies that the changes of the zero point energy in the shifted resp. rotated sectors are the same. Thus, the contribution of the twist field and of the fractional oscillator numbers resulting from the twisted oscillators to the zero point energy is identical to $\frac{1}{2} \delta^{2}$ coming from the shifted states. Therefore, the twist fields correspond to vertex operators of the form $\exp i(\delta+p) \cdot \boldsymbol{X}$. We also understand that for a rotation which is equivalent to a shift, the rank of the gauge group is not reduced.

Although any Weyl rotation is equivalent to a shift in the lattice, there are many shifts which cannot be represented as a Weyl rotation. This is true especially for shifts with infinite order which nevertheless could lead to consistent string theories. E.g., concerning the left-moving part $\Gamma_{22}$, there is no need to consider shifts which correspond to Weyl rotations since superconformal invariance give no restrictions for this case. On the other hand, outer automorphisms like permutation symmetries of different lattice factors cannot be represented as shifts and will, in general, lead to theories with reduced rank of the gauge group. We will discard this case in the next chapter and restrict ourselves to inner automorphisms in order to keep contact with self-dual lattices.

Finally, let us again emphasize that the equivalence between shifts and rotations is only true for specific background parameters of the underlying torus such that the symmetry of $\Lambda$ is also realized on the momentum lattice $\Gamma_{22 ; 6}$. For arbitrary choices of background a Weyl rotation will reduce the rank of the gauge group [18] as will be seen in one of the examples.

\section{Covariant lattices}

Four-dimensional heterotic strings in the covariant lattice approach [4, 10, 21-25] are obtained by combining the momentum lattice of the compactified bosons $\Gamma_{22 ; 6}$ with the weight lattice $\Gamma_{5,1}$ of the bosonized right-moving world sheet fermions $\psi^{\mu}$ and the bosonized superconformal ghosts $\beta, \gamma$. The signature of this lattice is 
$\left((+1)^{5}(-1)\right)$ where the last entry of each lattice vector corresponds to the ghost charge $q$ of the state considered. Then the 4-dimensional heterotic string theory is characterized by a lattice $\Gamma_{22 ; 11,1}=\Gamma_{22 ; 6} \otimes \Gamma_{5,1}$. Modular invariance enforces $\Gamma_{22 ; 11,1}$ to be an odd self-dual lorentzian lattice. Vectors of this lattice are denoted by $\boldsymbol{p}=\left(\boldsymbol{p}_{\mathrm{L}}, \boldsymbol{p}_{\mathrm{R}}, q\right)$. The corresponding string states have the following mass:

$$
\begin{aligned}
\frac{1}{8} m_{\mathrm{L}}^{2} & =\frac{1}{2} \boldsymbol{p}_{\mathrm{L}}^{2}+N_{\mathrm{L}}-1, \\
\frac{1}{8} m_{\mathrm{R}}^{2} & =\frac{1}{2} p_{\mathrm{R}}^{2}-\frac{1}{2} q^{2}-q+N_{\mathrm{R}}-1, \\
m^{2} & =\frac{1}{2}\left(m_{\mathrm{L}}^{2}+m_{\mathrm{R}}^{2}\right), \quad m_{\mathrm{L}}^{2}=m_{\mathrm{R}}^{2} .
\end{aligned}
$$

The torus compactification corresponds to the case that the $\Gamma_{22 ; 6}$ part is even self-dual and $\Gamma_{5,1}$ is an odd self-dual lattice. Then $\Gamma_{5,1}$ has to be the weight lattice of $\mathrm{D}_{5,1}$ with 0 and $\mathrm{S}$ conjugacy classes. It corresponds to the superstring lattice in 10 dimensions [21,33] or equivalently in 4 dimensions to the $N=4$ supergravity model. This can be seen by decomposing $\Gamma_{5,1}$ into $\mathrm{D}_{3} \otimes \mathrm{D}_{2,1}$ where $\mathrm{D}_{2,1}$ describes the 4-dimensional Lorentz group SO(4) plus ghost excitations. States in the canonical ghost picture have fixed ghost charge: $q=-\frac{1}{2}$ for space-time fermions and $q=-1$ for space-time bosons. The spinor conjugacy class of $D_{5,1}$ is decomposed as $(\mathrm{S}, \mathrm{S})+(\mathrm{C}, \mathrm{C})$ under $\mathrm{D}_{3} \otimes \mathrm{D}_{2,1}$ and leads to 4 gravitinos in 4 dimensions. Furthermore, we recognize that any fermion state $\mathrm{S}$ of $\mathrm{SO}(4)_{\text {Lorentz }}$ is accompanied by its chiral partner $\mathrm{C}$, i.e. there is no chiral fermion spectrum.

Instead of the lorentzian lattice $\Gamma_{5,1}$, it is more convenient to consider an euclidean lattice $\Gamma_{8}$ which is obtained by changing the dimension of $\Gamma_{5,1}$ by 4 units keeping all conjugacy classes the same (see [4,22]). Then the lattice $\Gamma_{22 ; 14}=\Gamma_{22 ; 6} \otimes \Gamma_{8}$ must be even self-dual. For the torus compactification $\Gamma_{8}$ must be even self-dual, i.e. the root lattice of $\mathrm{E}_{8}$. Thus all $N=4$ supersymmetric theories in four dimensions are characterized by the $E_{8}$ root lattice with spinor and 0 conjugacy classes of $D_{8}$. Thus, in order to break the $N=4$ supersymmetry and to get chiral fermions, one has to consider combined shifts or twists in $\Gamma_{22 ; 6}$ and $\mathrm{E}_{8}$ which correspond to generalized GSO projections in the compactified as well as fermionic sector of the theory.

Thus, let us consider an orbifold where we mod out a translational symmetry. This modding is realized by a shift which acts simultaneously in $\Gamma_{22 ; 6}$ and $\mathrm{E}_{8}$ such that the shifted lattice $\Gamma_{22 ; 14}^{\prime}=\Gamma_{22 ; 6}^{\prime} \otimes \Gamma_{8}^{\prime}$ is again self-dual, however not the sublattices $\Gamma_{22 ; 6}^{\prime}$ and $\Gamma_{8}^{\prime}$. Then it is possible to obtain a theory with chiral fermions and $N=1$ or $N=0$ supersymmetry as we will see later.

First, we want to prove that the shifted lattice is again self-dual leading to a modular invariant theory. Suppose we are starting from a torus compactification described by an even lorentzian self-dual lattice $\Gamma \equiv \Gamma_{22 ; 14}=\Gamma_{22 ; 6} \otimes \mathrm{E}_{8}$. The lattice vectors of $\Gamma$ are denoted by $\boldsymbol{p}=\left(\boldsymbol{p}_{\mathrm{L}}, \boldsymbol{p}_{\mathrm{R}}\right)$ with scalar product $\boldsymbol{p}^{2}=-\boldsymbol{p}_{\mathrm{L}}^{2}+\boldsymbol{p}_{\mathrm{R}}^{2}$. Let 
us consider a shift of order $\mathrm{N}$ generated by a shift vector $\delta=\left(\delta_{\mathrm{L}}, \delta_{\mathrm{R}}\right)$ with $N \delta \in \Gamma$. It can be shown that there exist a lattice vector $\tilde{\boldsymbol{p}} \in \Gamma$ and $\delta \cdot \tilde{\boldsymbol{p}}=1 / N \bmod 1$. Then the self-dual lattice $\Gamma$ can be represented as the union (direct sum) of "conjugacy classes"

$$
\Gamma=\Gamma_{0} \oplus \Gamma_{1} \oplus \Gamma_{2} \oplus \cdots \oplus \Gamma_{N-1}
$$

with

$$
\begin{gathered}
\boldsymbol{p}_{0} \in \Gamma_{0}, \quad \boldsymbol{p}_{0} \cdot \delta \in \mathrm{Z}, \\
\Gamma_{k}:=\Gamma_{0}+k \tilde{\boldsymbol{p}} .
\end{gathered}
$$

Thus, $\Gamma_{0}$ is itself a lattice whereas $\Gamma_{k}(k \neq 0)$ are not. Assume now that $\delta^{2}=$ $0 \bmod 2$ which automatically ensures the level matching condition eq. (2.12). The lattice $\Gamma^{\prime}=\Gamma_{22 ; 14}^{\prime}$, defined by

$$
\Gamma^{\prime}:=\Gamma_{0} \oplus \Gamma_{0}+\delta \oplus \Gamma_{0}+2 \delta \oplus \cdots \oplus \Gamma_{0}+(N-1) \delta,
$$

where $\Gamma_{0}$ corresponds to the unshifted sector and $\Gamma_{0}+\delta$ etc. to the various shifted sectors, is again an even lattice. Furthermore, all lattice vectors $\boldsymbol{p}_{0}+k \boldsymbol{\delta}, k=0, \ldots$, $N-1$ have integer scalar product since the original vectors $\boldsymbol{p} \in \Gamma$ had and $\boldsymbol{p}_{0} \cdot \boldsymbol{\delta} \in \mathrm{Z}$. Since on the other hand $\Gamma^{\prime}$ has the same number of conjugacy classes as $\Gamma$, there are no other conjugacy classes with vectors having integer scalar product with $\boldsymbol{p}^{\prime} \in \Gamma^{\prime}$. Thus, $\Gamma^{\prime}=\Gamma^{\prime *}$, i.e. $\Gamma^{\prime}$ is again an even self-dual lattice. Therefore, the orbifold which is obtained after modding out a translational symmetry from the enlarged lattice $\Gamma_{22 ; 14}$ is again described by an even self-dual covariant lattice.

Let us illustrate this briefly by the 10-dimensional (uncompactified) heterotic string theory. Take $\Gamma=\left(\mathrm{E}_{8} \otimes \mathrm{E}_{8}\right)_{\mathrm{L}} \otimes \mathrm{E}_{8 \mathrm{R}}$ leading to the supersymmetric $\mathrm{E}_{8} \times \mathrm{E}_{8}$ theory. $\Gamma$ can be written as $\Gamma=\left(\mathrm{D}_{8} \otimes \mathrm{D}_{8}\right)_{\mathrm{L}} \otimes \mathrm{D}_{8 \mathrm{R}}$ with the following 8 conjugacy classes: $(0,0,0),(0,0, S),(S, 0,0),(S, 0, S),(0, S, 0),(0, S, S),(S, S, 0)$ and $(S, S, S)$. Taking $\delta=\left(1,0^{7}, 1,0^{7} ;-\frac{1}{2},\left(\frac{1}{2}\right)^{7}\right) \in(\mathrm{V}, \mathrm{V}, \mathrm{C})$ we see that $2 \delta \in \Gamma$. Then $\Gamma_{0}$ is given by the following conjugacy classes: $(0,0,0),(\mathrm{S}, 0, \mathrm{~S}),(0, \mathrm{~S}, \mathrm{~S}),(\mathrm{S}, \mathrm{S}, 0)$. Constructing the shifted sector, the lattice $\Gamma^{\prime}$ is defined by the following conjugacy classes in addition to $\Gamma_{0}:(\mathrm{V}, \mathrm{V}, \mathrm{C}),(\mathrm{C}, \mathrm{V}, \mathrm{V}),(\mathrm{V}, \mathrm{C}, \mathrm{V}),(\mathrm{C}, \mathrm{C}, \mathrm{C}) . \Gamma^{\prime}$ is again even self-dual. The gauge symmetry is broken by the shift to $\mathrm{O}(16) \times \mathrm{O}(16)$ and the theory is no longer supersymmetric, i.e. the 10 -dimensional gravitino is projected out. $\Gamma^{\prime}$ describes exactly the covariant lattice [22] of the non-supersymmetric, tachyon-free $O(16) \times$ $\mathrm{O}(16)$ theory $[34,35]$.

Returning to 4 dimensions, we have seen that even self-dual lattices $\Gamma_{22 ; 14}$ are obtained from each other by shifting with a vector $\delta$ and projecting onto invariant plus shifted states. On the other hand, all lattices $\Gamma_{22 ; 14}$ are also connected by

^We are grateful to P. Vecsernyés for discussion on this point. 
Lorentz rotations with elements in $\mathrm{SO}(22,14)$. Both, Lorentz rotations and lattice shifts cannot be chosen arbitrarily, but must obey the constraints of superconformal invariance.

The supercurrent of the torus compactification with lattice $\Gamma_{22 ; 6} \otimes \Gamma_{8}$ was given in eq. (2.3). Bosonizing the RNS fermions it takes the following form:

$$
T_{\mathrm{F}} \sim \sum_{m=1}^{4} \partial X_{m} \exp \left(i t_{m} \cdot \boldsymbol{X}\right)+\sum_{i=1}^{6} \partial X_{i} \exp \left(i t_{i}^{\prime} \cdot \boldsymbol{X}\right)
$$

We decompose $\Gamma_{8}$ into $\mathrm{D}_{3} \otimes \mathrm{D}_{5}$ where $\mathrm{D}_{5}$ describes 4 bosonized RNS fermions $\psi^{\mathrm{m}}$, $(m=1, \ldots, 4)$ leading to the space-time Lorentz group $\mathrm{SO}(4)$, plus the bosonized superconformal ghost, and $\mathrm{D}_{3}$ the 6 internal fermions $\psi^{i}(i=1, \ldots, 6)$. The vectors $\boldsymbol{t}_{m}$ are the 4 vector weights of $\mathrm{D}_{2_{\text {Lorent }}{ }^{\prime}} \boldsymbol{t}_{m}=( \pm 1,0),(0, \pm 1)$, and $\boldsymbol{t}_{i}^{\prime}$ the 6 vector weights of $\mathrm{D}_{3_{\text {interal }}}, \boldsymbol{t}_{i}^{\prime}=( \pm 1,0,0)+$ permutations. Since $T_{\mathrm{F}} \cdot e^{\phi}$ describes the picture changing operator (see [23]), the following vectors must lie on the lattice $\Gamma_{22 ; 6} \otimes \mathrm{D}_{3}$ $\otimes \mathrm{D}_{5}: \boldsymbol{T}=\left(0 ; 0^{3} ; \boldsymbol{t}_{m}, 1,0,0\right)$ and $\boldsymbol{T}^{\prime}=\left(0 ; \boldsymbol{t}_{i}^{\prime} ; 0^{2}, 1,0,0\right)$ (the first entry belongs to $\Gamma_{22 ; 6}$, the next 3 entries to $\mathrm{D}_{3}$ and the last 5 to $\mathrm{D}_{5}$; the last 3 entries correspond to the fixed ghost charge +1$)$. More generally, superconformal invariance implies the existence of the $(0, \mathrm{~V}, \mathrm{~V})$ conjugacy class under $\Gamma_{22 ; 6} \otimes \mathrm{D}_{3} \otimes \mathrm{D}_{5}$. Thus, the symmetry of the root lattice of $D_{3} \otimes D_{5}$ is enlarged to $D_{8}$ and, furthermore, with the spinor conjugacy class, to the root lattice of $\mathrm{E}_{8}$. The existence of these constraint vectors of course still allows for arbitrary Lorentz rotations in $\Gamma_{22 ; 6}$ as discussed in the previous section. However, in order to destroy at least some of the 4 space-time supersymmetries and to get chiral fermions, we must break $E_{8}$ by some lattice shift. This means that some or all constraint vectors $\left(\boldsymbol{t}_{i}^{\prime} ; 0^{2}, 1,0,0\right)$ of $\mathrm{D}_{3} \otimes \mathrm{D}_{5}$ have to be projected out of the invariant spectrum and the supercurrent has to take a different form.

Let us decompose $\Gamma_{22 ; 14}=\Gamma_{22 ; 6} \otimes \Gamma_{8}$ in the following way

$$
\Gamma_{22 ; 14}=\left(\Gamma_{22}\right)_{\mathrm{L}} \otimes\left(\Gamma_{9} \otimes \Gamma_{5}\right)_{\mathrm{R}} .
$$

$\Gamma_{9}$ corresponds to 6 right moving compactified bosons and 6 bosonized internal fermions; $D_{5}$ again describes the 4-dimensional Lorentz group plus ghost contributions. Then, the supercurrent can be written in general $[10,23]$ :

$$
T_{\mathrm{F}} \sim \sum_{m=1}^{4} \partial X_{m} \exp \left(i t_{m} \cdot \boldsymbol{X}\right)+\sum_{\substack{\boldsymbol{t}^{\prime \prime} \in \Gamma_{9} \\\left(\boldsymbol{t}^{\prime \prime 2}=3\right)}} \exp \left(i \boldsymbol{t}^{\prime \prime} \cdot \boldsymbol{X}\right) .
$$

Again, the first term describes the space-time part of the supercurrent with $\boldsymbol{t}_{m}=$ $( \pm 1,0),(0, \pm 1)$, where the second part is the internal supercurrent. Combining $T_{\mathrm{F}}$ with $\mathrm{e}^{\phi}$ to obtain the picture changing operator, the lattice must contain, besides $\boldsymbol{T}$, the following constraint vectors: $T^{\prime \prime}=\left(0 ; \boldsymbol{t}^{\prime \prime} ; 0,0,1,0,0\right)$ (decomposition under $\Gamma_{22}$ $\left.\otimes \Gamma_{9} \otimes \mathrm{D}_{5}\right)$. Thus, $\boldsymbol{T}^{\prime \prime}$ replaces the "torus" constraint vector $\boldsymbol{T}^{\prime}$. 
For $\mathrm{D}_{n}$ lattices one can further specify $\boldsymbol{t}^{\prime \prime}[4,10,23]$. Decomposing $\Gamma_{9}$ into $\mathrm{D}_{1}$ factors, $\Gamma_{9}=\left(\mathrm{D}_{1}\right)^{9}$, the following vectors $t^{\prime \prime}$ must lie on the lattice:

$$
\boldsymbol{t}^{\prime \prime}=\left\{\begin{array}{l}
( \pm 1,0,0, \pm 1,0,0, \pm 1,0,0) \\
(0, \pm 1,0,0, \pm 1,0,0, \pm 1,0) \\
(0,0, \pm 1,0,0, \pm 1,0,0, \pm 1)
\end{array}\right.
$$

The last $3 \mathrm{D}_{1}$-components, e.g., correspond to the bosonized world sheet fermions.

Summarizing, in order to obtain chiral models the shifts must replace the original constraint vectors $\boldsymbol{T}^{\prime}$ of the torus compactification by the new constraint vectors $\boldsymbol{T}^{\prime \prime}$.

Let us now discuss the explicit form of the shift vectors breaking $\left(\mathrm{E}_{8}\right)_{\mathrm{R}}$ in order to obtain theories with $N=0,1,2,4$ supersymmetry.

For $N=4$ supersymmetry all gravitinos must survive. Thus, $\mathrm{E}_{8}$ must remain unbroken, i.e. no shifts in $\mathrm{E}_{8}$ are allowed. This means that all $N=4$ supersymmetric theories are just torus compactifications of the supersymmetric ten-dimensional heterotic string.

For theories with $N=2$ supersymmetry two gravitinos must be invariant under the shift. This means that $E_{8}$ must be broken to $E_{7} \times G$ where $G$ is a rank 1 simply laced group. The breaking $\mathrm{E}_{8} \rightarrow \mathrm{E}_{7} \times \mathrm{G}$ is realized by the following shift vector: $\delta=\left(a, a, 0^{6}\right)$. E.g. the $Z_{2}$ shift $\delta=\left(\frac{1}{2}, \frac{1}{2}, 0^{6}\right)$ breaks $\mathrm{E}_{8} \rightarrow \mathrm{E}_{7} \times \mathrm{SU}(2)$ where the $\mathrm{Z}_{3}$ shift $\delta=\left(\frac{1}{3}, \frac{1}{3}, 0^{6}\right)$ breaks $\mathrm{E}_{8} \rightarrow \mathrm{E}_{7} \times \mathrm{U}(1)$. The appearance of $\mathrm{E}_{7}$ guarantees $N=2$ supersymmetry [4]; the adjoint (root) representation of $E_{7}$ decomposes under $\mathrm{SU}(2) \times \mathrm{U}(1) \times \mathrm{SO}(10)$ as:

$$
\underline{133}=(\underline{3}, 0, \underline{1})+\left(\underline{2}, \frac{1}{2}, \underline{16}\right)+\left(\underline{2},-\frac{1}{2}, \underline{\overline{16}}\right)+(\underline{1}, 0, \underline{45})+(\underline{1}, 0, \underline{1})+(\underline{1}, \pm 1, \underline{10})
$$

(the second entry denotes the U(1) charge).

This root vector is always present in the lattice; the $\left(2, \frac{1}{2}, \underline{16}\right)$ part leads exactly to 2 gravitinos in 4 dimensions. Since $\mathrm{E}_{7}$ is not twisted, i.e. broken, the $N=2$ theories can be regarded as toroidal compactified 8-dimensional heterotic string theory which is itself obtained by an orbifold construction from 10 dimensions.

The 4-dimensional $N=1$ supersymmetric theories are realized if $\mathrm{E}_{8}$ gets broken to $E_{6} \times G$ where $G$ is a rank 2 simply laced group. The shift vector has the form $\boldsymbol{\delta}=\left(a, b, c, 0^{5}\right)$ with $\pm a \pm b \pm c=0$ (see [16]). E.g. the $Z_{3}$ shift $\delta=\left(\frac{1}{3}, \frac{1}{3},-\frac{2}{3}, 0^{5}\right)$ breaks $\mathrm{E}_{8}$ to $\mathrm{SU}(3) \times \mathrm{E}_{6}$, the $\mathrm{Z}_{4}$ shift $\delta=\left(\frac{1}{4}, \frac{1}{4},-\frac{1}{2}, 0^{5}\right) \mathrm{E}_{8} \rightarrow \mathrm{SU}(2) \times \mathrm{U}(1) \times \mathrm{E}_{6}$ and the $Z_{6}$ shift $\delta=\left(\frac{1}{6}, \frac{1}{3},-\frac{1}{2}, 0^{5}\right) E_{8} \rightarrow[U(1)]^{2} \times E_{6}$. Furthermore, these 3 examples can be equivalently realized as Weyl rotations in the $E_{8}$ root lattice. The appearance of the $\mathrm{E}_{6}$-root vectors is responsible for $N=1$ supersymmetry. The decomposition of the adjoint (root) of $\mathrm{E}_{6}$ to $\mathrm{U}(1) \times \mathrm{SO}(10)$ reads:

$$
\underline{78}=(0, \underline{45})+(0, \underline{1})+\left(-\frac{1}{2} \sqrt{3}, \underline{16}\right)+\left(\frac{1}{2} \sqrt{3}, \underline{16}\right)
$$


Thus the root vector leads model-independently to a "lattice vector" building the $\left(-\frac{1}{2} \sqrt{3}, 16\right)$ representation of $\mathrm{U}(1) \times \mathrm{SO}(10)$ giving rise to exactly one (left-handed) gravitino field. Furthermore, the $\mathrm{E}_{6}$ weights give rise to the chiral superfield structure of the matter fields in the 4-dimensional theory. Consider the fundamental representation of $\mathrm{E}_{6}$ :

$$
\underline{27}=\left(-\frac{1}{3} \sqrt{3}, \underline{10}\right)+\left(\frac{1}{6} \sqrt{3}, \underline{16}\right)+\left(\frac{2}{3} \sqrt{3}, \underline{1}\right) .
$$

These 3 representations correspond exactly to the 3 component fields of a chiral superfield in 4 dimensions. The first representation leads to the vertex operators of massless scalars in the ghost picture $q=-1$, the second representation to massless (chiral) fermions with $q=-\frac{1}{2}$ and the third representation to vertex operators of the scalar auxiliary F-fields (see also [36]), however in the ghost picture with $q=0$.

Finally, the non-supersymmetric $(N=0)$ theories are obtained by totally twisting 6 bosonized fermions with the following shift vector: $\delta=\left(a, b, c, 0^{5}\right), a, b, c \neq 0$, $\pm a \pm b \pm c \neq 0$. Then, $\mathrm{E}_{8}$ gets broken to $\mathrm{D}_{5} \times \mathrm{G}$ where $\mathrm{G}$ is a rank 3 simply laced group. Now, all gravitinos are projected out. E.g. the shift $\delta=\left(\frac{1}{2}, \frac{1}{2}, \frac{1}{2}, 0^{5}\right)$ breaks $\mathrm{E}_{8} \rightarrow \mathrm{SO}(6) \times \mathrm{SO}(10)$.

The covariant lattices are not only useful to understand the number of gravitinos in 4 dimensions but also to explain the number of (chiral) fermions in complex representations of the left moving gauge group. In the orbifold approach the degeneracy of fermions in the twisted sector is given by the number of fixed points in the 6 internal dimensions (see eqs. (2.19), (2.20) and (2.21)). However, in the covariant lattice approach the notion of fixed points is totally lost. Here $N_{\mathrm{F}}$ is in general determined by the transformation properties of the fermions under some part of the local group $G_{L}$ and also under the global symmetry group $G_{R}$ which is connected to the right moving ungauged part of the covariant lattice. It means that the number of fermion families is essentially given by the number of conjugacy classes of the lattice containing representations leading to massless fermions. The number of conjugacy classes $N_{\mathrm{C}}$ of the self-dual lattice $\Gamma_{m, n}=\mathrm{G}_{\mathrm{L}} \times \mathrm{G}_{\mathrm{R}}$ is given by

$$
N_{\mathrm{C}}=\sqrt{\left(\text { order of center of } \mathrm{G}_{\mathrm{L}}\right) \times\left(\text { order of center of } \mathrm{G}_{\mathrm{R}}\right)} .
$$

Thus this formula reproduces eq. (2.21) for $G_{L}=G_{R}$ and establishes the equivalence between both approaches.

Furthermore, the texture of the Yukawa coupling matrix is determined for the orbifolds by the condition that the center-of-mass coordinates of the twisted states sum up to zero. This corresponds in the covariant lattice picture to the invariance of the Yukawa couplings under $G_{L} \times G_{R}$. Thus the Yukawa coupling matrix is determined by Clebsch-Gordan coefficients of $G_{L}, G_{R}$. Concluding these arguments, group theoretical properties, which are connected with the enhanced symmetries of the covariant lattices, correspond to topological properties of more general 
manifolds like the Euler number or intersection matrices [37]. The more general conformal field theories resp. manifolds lead in general to theories with rank $<22$ gauge symmetries. Therefore, given a specific covariant lattice and identifying it with the corresponding orbifold or even Calabi-Yau manifold, one is able to determine the part of the gauge symmetries which can be broken by varying the background parameters continuously. Thus, the masses of some string states, gauge particles, scalars and also fermions, can vary smoothly and are zero only for specific background parameters. Of course, the chiral structure of the theory cannot be changed by smooth background variation. Therefore, some of the gauge symmetries may not be affected at all by the choice of background parameters.

Finally, we like to discuss the vertex operators which appear in the 4-dimensional models. For the case of orbifolds obtained after modding out a rotational symmetry, states in the untwisted sector are created by oscillators with integer oscillator number or by operators of the form $\exp \left(i \boldsymbol{p}_{0} \cdot \boldsymbol{X}\right)$ where $\boldsymbol{p}_{0}$ is a vector in the untwisted lattice $\Gamma_{0}$. States in the twisted sector are described by twist fields and oscillators with non-integer oscillator number.

On the other hand, in the covariant lattice approach every vertex operator can be expressed in terms of free bosonic fields. This means that certain vertex operators provide an explicit realization of twist fields with the same conformal dimension. Specifically, vertex operators which describe excitations in the 6-dimensional sublattice $\Gamma_{6 ; 6}$, originating from the compactified bosons, are equivalent to the twist fields of the orbifold. It is worth mentioning that the twist fields which appear in this context always correspond to the simultaneous twisting of two fermions in the fermionic description since the free bosons building the Cartan subalgebra of the covariant lattice have periodic boundary conditions. For $\mathrm{D}_{n}$ models, one can conclude therefore that the minimal conformal dimension of a twist-field is $1 / 8$ corresponding to a vertex operator $\exp \left(i \frac{1}{2} X\right)$ where $X$ is a single bosonic field.

In the same way, states with non-integer oscillator number correspond to vertex operators with lattice vectors in the internal dimensions.

\section{Examples}

In this section we would like to illustrate the previous discussion by two explicit examples. The first model is the well-known $Z_{3}$ orbifold [16].

Let us start with the torus compactification of the 10-dimensional $E_{8} \times E_{8}$ heterotic string theory. The underlying 6-dimensional torus, the left- and right-moving bosonic coordinates are compactified on, is the product of three SU(3) root lattices. The six (length) ${ }^{2}=2$ roots of $\mathrm{SU}(3)$ are given by the following vectors:

$$
\begin{array}{ll}
\alpha_{1}=(\sqrt{2}, 0), \quad \alpha_{2}=\left(-\sqrt{\frac{1}{2}}, \sqrt{\frac{3}{2}}\right), & \alpha_{3}=(-\sqrt{2}, 0), \\
\alpha_{4}=\left(\sqrt{\frac{1}{2}},-\sqrt{\frac{3}{2}}\right), \quad \alpha_{5}=\left(\sqrt{\frac{1}{2}}, \sqrt{\frac{3}{2}}\right), & \alpha_{6}=\left(-\sqrt{\frac{1}{2}},-\sqrt{\frac{3}{2}}\right) .
\end{array}
$$


The momenta $\tilde{\boldsymbol{p}}_{\mathrm{L}}, \tilde{\boldsymbol{p}}_{\mathrm{R}}$, canonical to the compactified coordinates, are expressed by the winding vectors which are vectors in the $\mathrm{SU}(3)$ root lattice and also by the background parameters (see eq. (2.2)). By choosing the SU(3) root lattice for compactification, 12 elements of $g_{i j}$ are fixed. On the other hand, $B_{i j}$ and $A_{i}^{I}$ are still free parameters. For a generic choice of these parameters the gauge symmetry is $\mathrm{E}_{8} \times \mathrm{E}_{8} \times[\mathrm{U}(1)]^{6}$. However, for $A_{i}^{I}=0$ and $B_{i j}=\frac{1}{4}\left[\left(\begin{array}{cc}0 & -1 \\ 1 & 0\end{array}\right)\right]^{3}$ (see [29]) the lattice $\Gamma_{6,6}$ with lattice vectors $\tilde{\boldsymbol{p}}_{\mathrm{L}}, \tilde{\boldsymbol{p}}_{\mathrm{R}}$ is the even self-dual lorentzian weight lattice $[\mathrm{SU}(3)]_{\mathrm{L}}^{3} \otimes[\mathrm{SU}(3)]_{\mathrm{R}}^{3}$. Then, the gauge symmetry is enhanced to $\mathrm{E}_{8} \times \mathrm{E}_{8} \times[\mathrm{SU}(3)]_{\mathrm{L}}^{3}$ $\times[\mathrm{SU}(3)]_{\mathrm{R}}^{3}$. The general weight lattice $[\mathrm{SU}(3)]_{\mathrm{L}}^{3} \otimes[\mathrm{SU}(3)]_{\mathrm{R}}^{3}$ consists out of $3^{6}=729$ conjugacy classes since the order of the center of each $\mathrm{SU}(3)$ is 3 . We denote the 3 conjugacy classes of SU(3) by 0 (containing the roots) 3 and $\overline{3}$ according to their lowest dimensional representation $\underline{3}$ and $\underline{\overline{3}}$. The weights of $\underline{3}$ and $\underline{\overline{3}}$ are given by:

$$
\begin{array}{ll}
\text { 3: } & \omega_{1}=\left(\sqrt{\frac{1}{2}}, \sqrt{\frac{1}{6}}\right), \quad \omega_{2}=\left(-\sqrt{\frac{1}{2}}, \sqrt{\frac{1}{6}}\right), \quad \omega_{3}=\left(0,-\sqrt{\frac{2}{3}}\right), \\
\overline{3}: & \omega_{1}^{\prime}=\left(-\sqrt{\frac{1}{2}},-\sqrt{\frac{1}{6}}\right), \quad \omega_{2}^{\prime}=\left(\sqrt{\frac{1}{2}},-\sqrt{\frac{1}{6}}\right), \quad \omega_{3}^{\prime}=\left(0, \sqrt{\frac{2}{3}}\right) .
\end{array}
$$

To restrict the weight lattice to be self-dual $\Gamma_{6 ; 6}$ must contain only a subset of $\sqrt{729}=27$ conjugacy classes. These are:

$$
\begin{aligned}
& (0,0,0 ; 0,0,0) \\
& (3,3,3 ; 3,3,3) \\
& (\overline{3}, \overline{3}, \overline{3} ; \overline{3}, \overline{3}, \overline{3}) \\
& (0,0,3 ; 0,0,3)+\text { simultan. permut. of } 3 \\
& (0,3,3 ; 0,3,3)+\text { simultan. permut. of } 0 \\
& (0,0, \overline{3} ; 0,0, \overline{3})+\text { simultan. permut. of } \overline{3} \\
& (0, \overline{3}, \overline{3} ; 0, \overline{3}, \overline{3})+\text { simultan. permut. of } 0 \\
& (3,3, \overline{3} ; 3,3, \overline{3})+\text { simultan. permut. of } \overline{3} \\
& (3, \overline{3}, \overline{3} ; 3, \overline{3}, \overline{3})+\text { simultan. permut. of } 3 \\
& (0,3, \overline{3} ; 0,3, \overline{3})+\text { simultan. permut. of } 0,3, \overline{3} .
\end{aligned}
$$

This set of lattice vectors together with the roots of $\mathrm{E}_{8} \times \mathrm{E}_{8}$ defines the even self-dual lattice $\Gamma_{22 ; 6}=\left(\mathrm{E}_{8} \otimes \mathrm{E}_{8} \otimes[\mathrm{SU}(3)]^{3}\right)_{\mathrm{L}} \otimes[\mathrm{SU}(3)]_{\mathrm{R}}^{3}$. Finally, the covariant lattice $\Gamma_{22 ; 14}$ in four dimensions is just given by the direct product of $\Gamma_{22 ; 6}$ with the right moving root lattice of $\mathrm{E}_{8}$ coming from the bosonization of the RNS fermions 
as well as the superconformal ghosts. The $\mathrm{E}_{8}$ root lattice can be written as the following $D_{n}$ weight lattice: $\mathrm{E}_{8}=\mathrm{D}_{3} \otimes \mathrm{D}_{2} \otimes \mathrm{D}_{3}$. The first $\mathrm{D}_{3}$ factor (with lattice vectors $\boldsymbol{p}_{\mathrm{R}}^{\prime}$ ) corresponds to the internal bosonized world sheet fermions, the $\mathrm{D}_{2}$-factor (with lattice vectors $\boldsymbol{p}_{\mathrm{R}}$ ) to the space-time fermions generating states which build representations of the Lorentz group $\mathrm{SO}(4)$, and the last $\mathrm{D}_{3}$ factor (vectors $\boldsymbol{p}_{\mathrm{R}}^{\prime \prime}$ ) belongs to the ghost degrees of freedom.

The spectrum is easily worked out using the mass formula of eq. (3.2). The massless (non-abelian) gauge bosons correspond to lattice vectors $\boldsymbol{p}_{\mathrm{L}}^{2}=2, \tilde{\boldsymbol{p}}_{\mathrm{L}}^{2}=0$ or $\boldsymbol{p}_{\mathrm{L}}^{2}=0, \tilde{\boldsymbol{p}}_{\mathrm{L}}^{2}=2, \boldsymbol{p}_{\mathrm{L}}, \tilde{\boldsymbol{p}}_{\mathrm{L}} \in$ root of $\mathrm{G}_{\mathrm{L}}, \tilde{\boldsymbol{p}}_{\mathrm{R}}=\boldsymbol{p}_{\mathrm{R}}^{\prime}=\mathbf{0}, \boldsymbol{p}_{\mathrm{R}}=( \pm 1,0),(0, \pm 1)$ and $\boldsymbol{p}_{\mathrm{R}}^{\prime \prime}=$ $(1,0,0)$ (this choice corresponds to the canonical ghost picture, see [21]). The (left-handed) gauginos have $\boldsymbol{p}_{\mathrm{L}}^{2}$ or $\tilde{\boldsymbol{p}}_{\mathrm{L}}^{2}=2, \boldsymbol{p}_{\mathrm{L}}, \tilde{\boldsymbol{p}}_{\mathrm{L}} \in \operatorname{root}$ of $\mathrm{G}_{\mathrm{L}}, \tilde{\boldsymbol{p}}_{\mathrm{R}}=0, \boldsymbol{p}_{\mathrm{R}}^{\prime}=$ $\left( \pm \frac{1}{2}, \pm \frac{1}{2}, \pm \frac{1}{2}\right.$ ) (even number of “ - " signs), $\boldsymbol{p}_{\mathrm{R}}=\left(+\frac{1}{2},+\frac{1}{2}\right),\left(-\frac{1}{2},-\frac{1}{2}\right)$ and $\boldsymbol{p}_{\mathrm{R}}^{\prime \prime}=$ $\left(\frac{1}{2}, \frac{1}{2}, \frac{1}{2}\right)$. The four massless gravitinos are obtained if $N_{\mathrm{L}}=1, \boldsymbol{p}_{\mathrm{L}}=\tilde{\boldsymbol{p}}_{\mathrm{L}}=\tilde{\boldsymbol{p}}_{\mathrm{R}}=\mathbf{0}$, $\boldsymbol{p}_{\mathrm{R}}^{\prime}=\left( \pm \frac{1}{2}, \pm \frac{1}{2}, \pm \frac{1}{2}\right.$ ) (even number of “ - " signs), $\boldsymbol{p}_{\mathrm{R}}=\left(\frac{1}{2}, \frac{1}{2}\right),\left(-\frac{1}{2},-\frac{1}{2}\right), \boldsymbol{p}_{\mathrm{R}}^{\prime \prime}=$ $\left(\frac{1}{2}, \frac{1}{2}, \frac{1}{2}\right)$. In addition, there are massless scalars with $\boldsymbol{p}_{\mathrm{L}}^{2}$ or $\tilde{\boldsymbol{p}}_{\mathrm{L}}^{2}=2, \boldsymbol{p}_{\mathrm{L}}, \tilde{\boldsymbol{p}}_{\mathrm{L}} \in$ root of $\mathrm{G}_{\mathrm{L}}, \tilde{\boldsymbol{p}}_{\mathrm{R}}=0, \boldsymbol{p}_{\mathrm{R}}^{\prime}=( \pm 1,0,0)+$ permut., $\boldsymbol{p}_{\mathrm{R}}=\mathbf{0}, \boldsymbol{p}_{\mathrm{R}}^{\prime \prime}=(1,0,0)$. Thus, these scalars are 6-fold degenerate because of the non-trivial $D_{3_{R}}$ excitations. Of course, all states corresponding to root vectors of $[\mathrm{SU}(3)]_{\mathrm{L}}^{3}$ are only massless for the special $B_{i j}$ background chosen. Finally, there are massive states connected with non-trivial lattice vectors of $[\mathrm{SU}(3)]_{\mathrm{R}}^{3}$.

The symmetric $Z_{3}$ orbifold is constructed by modding out the $Z_{3}$ symmetry from the lattice $\Gamma_{22 ; 6} \otimes \mathrm{E}_{8}$.

First, we want to discuss briefly the case where the $Z_{3}$ symmetry is given as a simultaneous Weyl rotation $g$ on each factor of the $[\mathrm{SU}(3)]_{\mathrm{L}}^{3} \otimes[\mathrm{SU}(3)]_{\mathrm{R}}^{3}$ weight lattice. The $g$-action leaves $3^{3}=27$ fixed points invariant. Furthermore, one has to specify the twisting of the $\mathrm{E}_{8} \times \mathrm{E}_{8}$ gauge degrees of freedom. We have chosen the standard embedding, i.e. identifying a $\mathrm{SU}(3)$ subgroup of one of the $\mathrm{E}_{8}$ 's with the SU(3) subgroup of the SO(6) tangent group in the internal dimensions. This can be realized as a Weyl rotation in the $\mathbf{E}_{8}$ lattice or, equivalently, as a shift in this lattice. We restrict ourselves to the second possibility.

For the untwisted part of the spectrum one has to project onto states invariant under these rotations and shifts. First, three of the gravitinos are not invariant. Therefore, the theory is $N=1$ supersymmetric in four dimensions. The gauge symmetry $E_{8}$ gets broken to $E_{6} \times S U(3)$. For the case of arbitrary background parameters, the $[\mathrm{U}(1)]_{\mathrm{L}}^{6}$ gauge symmetry gets broken totally - the oscillator excitations are not invariant under the $Z_{3}$ rotation. However, if the gauge symmetry is enhanced to $[\mathrm{SU}(3)]_{\mathrm{L}}^{3}$ one recognizes that for each $\mathrm{SU}(3)$ there are two invariant linear combinations of massless "root" states:

$$
\begin{aligned}
& |1\rangle=\left|\alpha_{3}\right\rangle+\left|\alpha_{4}\right\rangle+\left|\alpha_{5}\right\rangle, \\
& |2\rangle=\left|\alpha_{1}\right\rangle+\left|\alpha_{2}\right\rangle+\left|\alpha_{6}\right\rangle .
\end{aligned}
$$


These states build the cartan subalgebra of two new U(1)'s such that the [SU(3)] $]_{\mathrm{L}}^{3}$ gauge symmetry is broken to $[\mathrm{U}(1)]_{\mathrm{L}}^{6}$. Therefore, the rank is not reduced in this case.

The chiral matter fields in the untwisted sector are fields transforming under the $(27,3)$ representation of the unbroken gauge group $\mathrm{E}_{6} \times \mathrm{SU}(3)$. These come in 3 copies since they are triplets under $\mathrm{SU}(3) \subset \mathrm{SO}(6)_{\text {tangent space. }}$. Finally, there are 3 chiral superfields which are singlets under $\mathrm{E}_{6} \times \mathrm{SU}(3)$. These also are 3-fold degenerate being triplets under the same SU(3). These 9 fields correspond to the freedom in choosing the still arbitrary metric parameters of the six-torus. For the case of the enhanced [SU(3)] ${ }^{3}$ gauge symmetry there are 18 additional massless chiral superfields. These correspond to the two invariant root combinations eq. (4.4) of each $\mathrm{SU}(3)$ again being triplets under $\mathrm{SU}(3) \subset \mathrm{SO}(6)_{\text {tangent space. }}$. For arbitrary background parameters these 18 particles, together with the 6 vector gauge fields of $[\mathrm{U}(1)]_{\mathrm{L}}^{6}$, become massive and realize the super Higgs mechanism. One recognizes that every vector particle is accompanied by 6 scalar fields which is in principle not necessary for the Higgs mechanism (see also [19]).

In the twisted sector one finds chiral superfields transforming under the $(27,1)$ and $3 \times(1, \overline{3})$ representation of $\mathrm{E}_{6} \times \mathrm{SU}(3)$. These are present at each of the 27 fixed points. Therefore, their degeneracy is 27 , and together with the untwisted sector one gets 36 families of 27's of $\mathrm{E}_{6}$ in this model.

The change of the zero point energy in the twisted sector is given with eq. (2.23) by $\frac{1}{3}$. This change in mass must be absorbed by the introduction of twist fields which creates the vacua in the twisted sectors. Therefore, the conformal dimensions of the twist fields is given by $\frac{1}{3}$.

Let us now obtain the $Z_{3}$ orbifold by representing the $Z_{3}$ symmetries entirely as translations, i.e. shifts on the covariant lattice. As pointed out before, the equivalence between the rotation and the shift in the $[\mathrm{SU}(3)]^{3}$ lattice is only true for the special $B_{i j}$ background. The torus compactification corresponds to the following covariant lattice:

$$
\Gamma_{22 ; 14}=\left[\mathrm{E}_{8} \otimes \mathrm{E}_{8} \otimes \mathrm{SU}(3)^{3}\right]_{\mathrm{L}} \otimes\left[\mathrm{SU}(3)^{3} \otimes \mathrm{E}_{8}\right]_{\mathrm{R}} .
$$

The $Z_{3}$ shift in one of the $E_{8_{1}}$ 's is given by:

$$
\boldsymbol{\delta}_{\mathrm{L}}=\left(\frac{1}{3}, \frac{1}{3},-\frac{2}{3}, 0^{5}\right) .
$$

This breaks $\mathrm{E}_{8} \rightarrow \mathrm{E}_{6} \times \mathrm{SU}(3)$. The simultaneous shifts in the $\mathrm{SU}(3)$ 's leading to the symmetric orbifold read:

$$
\tilde{\boldsymbol{\delta}}_{\mathbf{L}, \mathbf{R}}=\left(\frac{1}{3} \sqrt{\frac{1}{2}}, \sqrt{\frac{1}{6}}\right) .
$$

Thus, $\mathrm{SU}(3)$ is broken to $\mathrm{U}(1) \times \mathrm{U}(1)$.

Finally, the shift in the right moving $\mathrm{E}_{8}$ is again:

$$
\boldsymbol{\delta}_{\mathrm{R}}=\left(\frac{1}{3}, \frac{1}{3},-\frac{2}{3}, 0^{5}\right) \text {. }
$$


Again $E_{8}$ is broken to $S U(3) \times E_{6}$ which can be decomposed into $S U(3) \times U(1) \times$ $\mathrm{SO}(10) \supset \mathrm{SU}(3) \times \mathrm{U}(1) \times \mathrm{SO}(4) \times \mathrm{SO}(6)$. The $\mathrm{SU}(3) \times \mathrm{U}(1)$ part describes the fermionic degrees of freedom in the internal 6 dimensions, SO(4) the space-time Lorentz group and $\mathrm{SO}(6)$ the ghost excitations. As discussed in the last chapter, this shift vector breaks the $N=4$ supersymmetry to $N=1$ supersymmetry leaving only one gravitino invariant.

The combined $\mathrm{Z}_{3}$ shift vector on $\Gamma_{22 ; 14}$ has the following form:

$$
\delta=\left(\frac{1}{3}, \frac{1}{3},-\frac{2}{3}, 0^{5}, 0^{8},\left(\frac{1}{3} \sqrt{\frac{1}{2}}, \sqrt{\frac{1}{6}}\right)^{3} ;\left(\frac{1}{3} \sqrt{\frac{1}{2}}, \sqrt{\frac{1}{6}}\right)^{3}, \frac{1}{3}, \frac{1}{3},-\frac{2}{3}, 0^{5}\right),
$$

with the property $\delta^{2}=\delta_{\mathrm{L}}^{2}-\delta_{\mathrm{R}}^{2}=0$. Therefore using the argument of chapter 3 , the lattice $\Gamma_{22 ; 14}^{\prime}=\Gamma_{0} \oplus \Gamma_{0}+\delta \oplus \Gamma_{0}+2 \delta$ is again an even self-dual covariant lattice describing the $Z_{3}$ orbifold theory.

Let us first determine $\Gamma_{0}$, i.e. the untwisted sector with states invariant under the shift. For that purpose we classify all states according to their $Z_{3}$ eigenvalue under the shifts $\delta_{\mathrm{L}, \mathrm{R}}, \tilde{\boldsymbol{\delta}}_{\mathrm{L}, \mathrm{R}}$ (eqs. (4.6)-(4.8)). The 248 of $\mathrm{E}_{8}$ decomposes under $\mathrm{E}_{6} \times \mathrm{SU}(3)$ as follows where the subscript denotes the $\bar{Z}_{3}$ eigenvalue:

$$
\underline{248}=(\underline{78}, \underline{1})_{0}+(\underline{1}, \underline{8})_{0}+(\underline{27}, \underline{3})_{1 / 3}+(\underline{27}, \underline{3})_{2 / 3} .
$$

For the right-moving $E_{8}$ we have to decompose $E_{6}$ further to $U(1) \times D_{5}$ where $D_{5}$ describes the Lorentz group $\mathrm{SO}(4)$ plus ghost excitations (compare also with eqs. (3.10), (3.11)):

$$
\begin{aligned}
\underline{248}= & (\underline{1}, 0, \underline{45})_{0}+(\underline{1}, 0, \underline{1})_{0}+\left(\underline{1},-\frac{1}{2} \sqrt{3}, \underline{16}\right)_{0}+\left(\underline{1}, \frac{1}{2} \sqrt{3}, \underline{16}\right)_{0} \\
& +(\underline{8}, 0, \underline{1})_{0}+\left(\underline{3}, \sqrt{\frac{1}{12}}, \underline{16}\right)_{1 / 3}+\left(\underline{3},-\sqrt{\frac{1}{3}}, \underline{10}\right)_{1 / 3}+\left(\underline{3}, \frac{2}{3} \sqrt{3}, \underline{1}\right)_{1 / 3} \\
& +\left(\underline{\overline{3}},-\sqrt{\frac{1}{12}}, \underline{16}\right)_{2 / 3}+\left(\underline{\overline{3}}, \sqrt{\frac{1}{3}}, \underline{10}\right)_{2 / 3}+\left(\underline{\overline{3}},-\frac{2}{3} \sqrt{3}, \underline{1}\right)_{2 / 3} .
\end{aligned}
$$

The adjoint (roots) of $\mathrm{SU}(3)$ decompose under $\mathrm{U}(1) \times \mathrm{U}(1)$ as follows

$$
\begin{aligned}
\underline{8}= & 2(0,0)_{0}+(\sqrt{2}, 0)_{1 / 3}+\left(-\sqrt{\frac{1}{2}}, \sqrt{\frac{3}{2}}\right)_{1 / 3}+\left(-\sqrt{\frac{1}{2}},-\sqrt{\frac{3}{2}}\right)_{1 / 3} \\
& +(-\sqrt{2}, 0)_{2 / 3}+\left(\sqrt{\frac{1}{2}},-\sqrt{\frac{3}{2}}\right)_{2 / 3}+\left(\sqrt{\frac{1}{2}}, \sqrt{\frac{3}{2}}\right)_{2 / 3} .
\end{aligned}
$$

Finally the two inequivalent weights of $S U(3)$ have the $U(1) \times U(1)$ quantum numbers:

$$
\begin{aligned}
& \underline{3}=\left(-\sqrt{\frac{1}{2}}, \sqrt{\frac{1}{6}}\right)_{0}+\left(\sqrt{\frac{1}{2}}, \sqrt{\frac{1}{6}}\right)_{1 / 3}\left(0,-\sqrt{\frac{2}{3}}\right)_{2 / 3}, \\
& \underline{\overline{3}}=\left(\sqrt{\frac{1}{2}},-\sqrt{\frac{1}{6}}\right)_{0}+\left(0, \sqrt{\frac{2}{3}}\right)_{1 / 3}+\left(-\sqrt{\frac{1}{2}},-\sqrt{\frac{1}{6}}\right)_{2 / 3} .
\end{aligned}
$$


TABLE 1

Unshifted sector for the Z-orbifold

$\left[\begin{array}{lllllllllllll}\mathrm{E}_{6} & \mathrm{SU}(3) & \mathrm{E}_{8} & \mathrm{U}(1) & \mathrm{U}(1) & \mathrm{U}(1) & \mathrm{U}(1) & \mathrm{U}(1) & \mathrm{U}(1)]_{\mathrm{L}} & {\left[(\mathrm{U}(1))^{6}\right.} & \mathrm{SU}(3) & \mathrm{U}(1) & \mathrm{D}_{5}\end{array}\right]_{\mathrm{R}}$

\begin{tabular}{|c|c|c|c|c|c|c|c|c|c|c|c|c|c|}
\hline 1. & \multicolumn{3}{|c|}{1 and adjoint } & 0 & 0 & 0 & 0 & 0 & 0 & $0^{6}$ & $\underline{1}$ & 0 & 0 \\
\hline 2. & \multicolumn{3}{|c|}{$\underline{1}$ and adjoint } & 0 & 0 & 0 & 0 & 0 & 0 & $0^{6}$ & $\underline{1}$ & $\sqrt{\frac{3}{4}}$ & S \\
\hline \multirow[t]{10}{*}{3.} & $\underline{27}$ & $\underline{3}$ & $\underline{1}$ & 0 & 0 & 0 & 0 & 0 & 0 & $0^{6}$ & $\underline{3}$ & $-\sqrt{\frac{1}{3}}$ & V \\
\hline & $\underline{1}$ & $\underline{1}$ & $\underline{1}$ & $-\sqrt{2}$ & 0 & 0 & 0 & 0 & 0 & $0^{6}$ & $\underline{3}$ & $-\sqrt{\frac{1}{3}}$ & V \\
\hline & $\underline{1}$ & $\underline{1}$ & $\underline{1}$ & $-\sqrt{\frac{1}{2}}$ & $\sqrt{\frac{3}{2}}$ & 0 & 0 & 0 & 0 & $0^{6}$ & $\underline{3}$ & $-\sqrt{\frac{1}{3}}$ & V \\
\hline & $\underline{1}$ & $\underline{1}$ & $\underline{1}$ & $-\sqrt{\frac{1}{2}}$ & $-\sqrt{\frac{3}{2}}$ & 0 & 0 & 0 & 0 & $0^{6}$ & $\underline{3}$ & $-\sqrt{\frac{1}{3}}$ & $\mathrm{v}$ \\
\hline & $\underline{1}$ & 1 & $\underline{1}$ & 0 & 0 & $\sqrt{2}$ & 0 & 0 & 0 & $0^{6}$ & $\underline{3}$ & $-\sqrt{\frac{1}{3}}$ & $\mathrm{~V}$ \\
\hline & $\underline{1}$ & 1 & $\underline{1}$ & 0 & 0 & $-\sqrt{\frac{1}{2}}$ & $\sqrt{\frac{3}{2}}$ & 0 & 0 & $0^{6}$ & $\underline{3}$ & $-\sqrt{\frac{1}{3}}$ & $\mathrm{v}$ \\
\hline & $\underline{1}$ & $\underline{1}$ & $\underline{1}$ & 0 & 0 & $-\sqrt{\frac{1}{2}}$ & $-\sqrt{\frac{3}{2}}$ & 0 & 0 & $0^{6}$ & $\underline{3}$ & & V \\
\hline & $\underline{1}$ & $\underline{1}$ & $\underline{1}$ & 0 & 0 & 0 & 0 & $\sqrt{2}$ & 0 & $0^{6}$ & $\underline{3}$ & $-\sqrt{\frac{1}{3}}$ & V \\
\hline & $\underline{1}$ & $\underline{1}$ & $\underline{1}$ & 0 & 0 & 0 & 0 & $-\sqrt{\frac{1}{2}}$ & $\sqrt{\frac{3}{2}}$ & $0^{6}$ & $\underline{3}$ & $-\sqrt{\frac{1}{3}}$ & v \\
\hline & $\underline{1}$ & $\underline{1}$ & $\underline{1}$ & 0 & 0 & 0 & 0 & $-\sqrt{\frac{1}{2}}$ & $-\sqrt{\frac{3}{2}}$ & $0^{6}$ & $\underline{3}$ & $-\sqrt{\frac{1}{3}}$ & $\mathrm{~V}$ \\
\hline \multirow[t]{2}{*}{4.} & $\underline{27}$ & $\underline{3}$ & $\underline{1}$ & 0 & 0 & 0 & 0 & 0 & 0 & $0^{6}$ & $\underline{3}$ & $+\sqrt{\frac{1}{12}}$ & $\mathrm{~S}$ \\
\hline & $\underline{1}$ & $\underline{1}$ & $\underline{1}$ & \multicolumn{6}{|c|}{ same charges as in 3} & $0^{6}$ & $\underline{3}$ & $+\sqrt{\frac{1}{12}}$ & $\mathrm{~S}$ \\
\hline
\end{tabular}

Requiring invariance under the combined shift $\delta$ (eq. 4.9) we obtain the massless invariant spectrum as displayed in table 1 . Lattice vectors in the first row of this table correspond to gauge bosons of the rank 22 gauge group $\mathrm{E}_{6} \times \mathrm{SU}(3) \times \mathrm{E}_{8} \times$ $(\mathrm{U}(1))^{6}$, to gravitons, $B_{\mu \nu}$ and dilaton fields. In the second row, the supersymmetric fermionic partners appear. The third and the forth row correspond to chiral bosonic and fermionic matter fields. Note that one obtains the same number of states compared to the "rotational" construction of the $Z_{3}$ orbifold.

To construct the "shifted part" of the covariant lattice $\Gamma_{22 ; 14}^{\prime}$ we consider the twisted, i.e. shifted states of the theory which are built by lattice vectors $\boldsymbol{p}_{0}+\boldsymbol{\delta}$, $p_{0}+2 \delta$. First, let us again discuss the single parts of the lattice. Considering lattice vectors $\boldsymbol{p}_{0}+\boldsymbol{\delta}_{\mathrm{L}}$ with $\boldsymbol{p}_{0} \in$ root of $\mathrm{E}_{8}$ we obtain 27 states $\boldsymbol{p}^{\prime}$ with $\boldsymbol{p}^{\prime 2}=\frac{4}{3}$ building the $(27,1)$ representation of $\mathrm{E}_{6} \times \mathrm{SU}(3)$. Furthermore, we get 3 states with $\boldsymbol{p}^{\prime 2}=\frac{2}{3}$ transforming as $(\underline{1}, \underline{3})$ under $\mathrm{E}_{6} \times \mathrm{SU}(3)$.

Switching now to $\mathrm{SU}(3)$, the shift $\tilde{\boldsymbol{\delta}}_{\mathrm{L}, \mathrm{R}}$ leads to 3 states with $\tilde{\boldsymbol{p}}^{\prime 2}=\frac{2}{9}$, namely $\mathbf{0}+\boldsymbol{\delta}$ transforming under $\mathrm{U}(1) \times \mathrm{U}(1)$ as $\left(\frac{1}{3} \sqrt{\frac{1}{2}}, \sqrt{\frac{1}{6}}\right), \omega_{3}+\boldsymbol{\delta}=\left(\frac{1}{3} \sqrt{\frac{1}{2}},-\frac{1}{2} \sqrt{\frac{2}{3}}\right)$ and $\boldsymbol{\omega}_{1}^{\prime}+\boldsymbol{\delta}=\left(-\frac{2}{3} \sqrt{\frac{1}{2}}, 0\right)$. In addition, we obtain 3 states with $\tilde{\boldsymbol{p}}^{\prime 2}=\frac{8}{9}: \boldsymbol{\alpha}_{6}+\boldsymbol{\delta}=\left(-\frac{2}{3} \sqrt{\frac{1}{2}}\right.$, $\left.-\sqrt{\frac{2}{3}}\right), \omega_{2}+\delta=\left(-\frac{2}{3} \sqrt{\frac{1}{2}}, \sqrt{\frac{2}{3}}\right)$ and $\omega_{2}^{\prime}+\delta=\left(\frac{4}{3} \sqrt{\frac{1}{2}}, 0\right)$. Thus looking at the combined $[\mathrm{U}(1)]^{6}$ lattice, there are 27 shifted states with $\tilde{\boldsymbol{p}}^{\prime 2}=\frac{2}{3}$ coming from the 27 [SU(3)] ${ }^{3}$ 
TABLE 2

Shifted sector for the Z-orbifold

\begin{tabular}{ccccccccc}
\hline & {$\left[\mathrm{E}_{6}\right.$} & $\mathrm{SU}(3)$ & $\mathrm{E}_{8}$ & $\left.(\mathrm{U}(1))^{6}\right]_{\mathrm{L}}$ & {$\left[(\mathrm{U}(1))^{6}\right.$} & $\mathrm{SU}(3)$ & $\mathrm{U}(1)$ & $\left.\mathrm{D}_{5}\right]_{\mathrm{R}}$ \\
\hline 1. & 27 & $\underline{1}$ & $\underline{1}$ & $\begin{array}{c}27 \text { combinations } \\
\text { with } \tilde{\boldsymbol{p}}^{\prime 2}=\frac{2}{3}\end{array}$ & $\begin{array}{c}\text { same } 27 \\
\text { combinations }\end{array}$ & $\underline{1}$ & $\sqrt{\frac{1}{12}}$ & $\mathrm{~S}$ \\
2. & $\underline{27}$ & $\underline{1}$ & $\underline{1}$ & $"$ & $\prime$ & $\underline{1}$ & $\sqrt{\frac{1}{3}}$ & $\mathrm{~V}$ \\
3. & $\underline{1}$ & $\underline{3}$ & $\underline{1}$ & $\begin{array}{c}81 \text { combinations } \\
\text { with } \tilde{\boldsymbol{p}}^{\prime 2}=\frac{4}{3}\end{array}$ & $\prime \prime$ & $\underline{1}$ & $\sqrt{\frac{1}{12}}$ & $\mathrm{~S}$ \\
4. & $\underline{1}$ & $\underline{\underline{3}}$ & $\underline{1}$ & $"$ & $\prime$ & $\underline{1}$ & $\sqrt{\frac{1}{3}}$ & $\mathrm{~V}$ \\
\hline
\end{tabular}

conjugacy classes of eq. (4.3). Furthermore, there are 81 additional states with $\tilde{\boldsymbol{p}}^{\prime 2}=4 / 3$ where each of the 27 conjugacy classes of $[\mathrm{SU}(3)]^{3}$ leads to 3 of these shifted states.

Finally let us discuss the shifted states resulting from the right moving $\mathrm{E}_{8}$. Here we get 2 states with $\boldsymbol{p}^{\prime 2}=\frac{4}{3}$ transforming under $\mathrm{SU}(3) \times \mathrm{U}(1) \times \mathrm{SO}(10)$ as $\left(1, \sqrt{\frac{1}{12}}, 16\right)$ and $\left(1,-\sqrt{\frac{1}{3}}, 10\right)$. Now, collecting all these different pieces, we obtain the mass spectrum in the shifted sector given in table 2. First, we have chiral fermions plus their bosonic partners in the $(27,1)$ representation of $\mathrm{E}_{6}$. Their degeneracy is given by the different $[\mathrm{U}(1)]_{\mathrm{L}, \mathrm{R}}^{6}$ quantum numbers. There are 27 possible different combinations since the original self-dual lattice $[\mathrm{SU}(3)]_{\mathrm{L}}^{3} \otimes$ $[\mathrm{SU}(3)]_{\mathrm{R}}^{3}$ possesses 27 conjugacy classes.

Analogously, we obtain 81 fermionic plus bosonic states transforming as $(1, \overline{3})$ under $\mathrm{E}_{6} \times \mathrm{SU}(3)$. Here one specific conjugacy class of $[\mathrm{SU}(3)]_{\mathrm{L}}^{3}$ like $(3,3,3)$ leads to 3 possible $[\mathrm{U}(1)]_{\mathrm{L}}^{6}$ combinations since we have to demand for masslessness that $\tilde{\boldsymbol{p}}_{\mathrm{L}}^{\prime 2}=\frac{4}{3}$ and $\tilde{\boldsymbol{p}}_{\mathrm{R}}^{\prime 2}=\frac{2}{3}$.

One can now easily verify that the newly obtained lattice $\Gamma_{22,14}^{\prime}=\Gamma_{0} \oplus \Gamma_{0}+\delta \oplus \Gamma_{0}$ $+2 \delta$ is in fact even self-dual. The scalar products between states in the shifted and unshifted sector are integer. Furthermore, together with the massive states not displayed in tables 1 and 2 the number of conjugacy classes of $\Gamma_{22 ; 14}$ and $\Gamma_{22 ; 14}^{\prime}$ is the same, i.e. the lattice is self-dual.

Let us now illustrate the general statements of chapter 2, 3 about the chiral structure and twist fields of the theory. In the covariant lattice approach, the number of chiral fermions is determined by the number of possible charge combinations of $(\mathrm{U}(1))^{6}$. These originate from the $27[\mathrm{SU}(3)]_{\mathrm{L}}^{3} \otimes[\mathrm{SU}(3)]_{\mathrm{R}}^{3}$ conjugacy classes (cf. eq. (3.12)) which leads to 27 families of $(27,1)$ of $\mathrm{E}_{6} \times \mathrm{SU}(3)$. This number is identical to the number of fixed points (cf. eq. 2.21 ) in the case where one mods out by the rotational $Z_{3}$ symmetry.

Due to the lattice interpretation of the twisted sector, we are able to give an explicit realization of the 27 twist fields which are needed to create the 27 twisted ground states at each of the fixed points. These are given in terms of lattice vectors 
of the $[\mathrm{U}(1)]_{\mathrm{L}}^{6} \otimes[\mathrm{U}(1)]_{\mathrm{R}}^{6}$ lattice whose components are simply the $\mathrm{U}(1)$ charges. For example, the twist vertex operator corresponding to the state $\left(\tilde{\delta}_{\mathrm{L}}^{3}, \tilde{\delta}_{\mathrm{R}}^{3}\right)$ is given by the following expression:

$$
\begin{aligned}
\sigma(\bar{z}, z) & =\exp \left[i\left(\frac{1}{3} \sqrt{\frac{1}{2}} \tilde{X}_{1}+\sqrt{\frac{1}{6}} \tilde{X}_{2}+\frac{1}{3} \sqrt{\frac{1}{2}} \tilde{X}_{3}+\sqrt{\frac{1}{6}} \tilde{X}_{4}+\frac{1}{3} \sqrt{\frac{1}{2}} \tilde{X}_{5}+\sqrt{\frac{1}{6}} \tilde{X}_{6}\right)\right](\bar{z}) \\
& \times \exp \left[i\left(\frac{1}{3} \sqrt{\frac{1}{2}} \tilde{X}_{1}+\sqrt{\frac{1}{6}} \tilde{X}_{2}+\frac{1}{3} \sqrt{\frac{1}{2}} \tilde{X}_{3}+\sqrt{\frac{1}{6}} \tilde{X}_{4}+\frac{1}{3} \sqrt{\frac{1}{2}} \tilde{X}_{5}+\sqrt{\frac{1}{6}} \tilde{X}_{6}\right)\right](z),
\end{aligned}
$$

where the $\tilde{X}_{i}(i=1 \ldots 6)$ are the internal compactified bosons. Analogously there exist further 26 vertex operators of this type. All of these have the conformal dimension $h=\frac{1}{2} \tilde{\boldsymbol{p}}_{\mathrm{L}}^{\prime 2}=\bar{h}=\frac{1}{2} \tilde{\boldsymbol{p}}_{\mathrm{R}}^{\prime 2}=\frac{1}{3}$. The same conformal dimension was also computed for the twist field in the last paragraph. Calculating the Yukawa amplitudes using these vertex operators, one obtains selection rules such that the amplitudes are invariant under $[\mathrm{U}(1)]_{\mathrm{L}}^{6} \otimes[\mathrm{U}(1)]_{\mathrm{R}}^{6}$. These selection rules reproduce exactly the ones obtained by requiring that the center-of-mass coordinates of the twisted states add up to zero.

Finally, in the orbifold construction, there are also states with non-integer oscillator number. For example, the $(1, \overline{3})$ matter fields under $\mathrm{E}_{6} \times \mathrm{SU}(3)$ in the twisted sector are created by vertex operators $\partial X^{i}(\bar{z}) / \partial \bar{z}^{1 / 3}$ with conformal dimension $1 / 3$ acting on the twisted ground state [31]. This leads to an extra degeneracy factor of 3 . Again we can translate this feature into the covariant lattice description. Here the degeneracy of these states comes from the 81 lattice vectors of $[\mathrm{U}(1)]_{\mathrm{L}}^{6}$ with $\tilde{\boldsymbol{p}}_{\mathrm{L}}^{\prime 2}=\frac{4}{3}$. Thus, these states are created by operators $\exp \left(i \tilde{\boldsymbol{p}}_{\mathrm{L}}^{\prime} \cdot \boldsymbol{X}(\vec{z})\right)$ with $\tilde{\boldsymbol{p}}_{\mathrm{L}}^{\prime} \in$ $[\mathrm{U}(1)]_{\mathrm{L}}^{6}, \tilde{\boldsymbol{p}}_{\mathrm{L}}^{\prime 2}=\frac{4}{3}$.

Our next, more briefly discussed example is an asymmetric $\mathrm{Z}_{4}$ orbifold which is described by a $D_{n}$-covariant lattice. This model was already discussed in $[4,23]$. It has no space-time supersymmetry, is tachyon-free and possesses chiral fermions. Unlike the previous example this model can no longer be understood as a compactification of the 10-dimensional heterotic string theory.

The underlying self-dual lattice of the torus compactification is given by the weight lattice of:

$$
\Gamma_{22 ; 6}=\left[\mathrm{D}_{10} \otimes \mathrm{D}_{5} \otimes \mathrm{D}_{1} \otimes \mathrm{D}_{2} \otimes \mathrm{D}_{4}\right]_{\mathrm{L}} \otimes\left[\mathrm{D}_{6}\right]_{\mathrm{R}} .
$$

It contains 64 conjugacy classes like

$$
\begin{aligned}
& p_{1} \simeq(0, S, C, S, 0 ; 0), \\
& p_{2} \simeq(0, S, C, S, C ; V), \\
& p_{3} \simeq(C, S, C, S, V ; S), \\
& p_{4} \simeq(0, V, V, 0,0 ; 0), \\
& p_{5} \simeq(0,0, V, V, 0 ; 0), \\
& p_{6} \simeq(0, V, 0, V, 0 ; 0) .
\end{aligned}
$$


Therefore, the massless matter states of the theory transform under $G_{L}=\operatorname{SO}(20) \times$ $\mathrm{SO}(10) \times \mathrm{SO}(2) \times \mathrm{SO}(4) \times \mathrm{SO}(8)$ as

$$
\begin{aligned}
& \left(\underline{1}, \underline{16},-\frac{1}{2}, \underline{2}, \underline{1}\right), \\
& (\underline{1}, \underline{10}, 1, \underline{1}, \underline{1}), \\
& (\underline{1}, \underline{1}, 1, \underline{4}, \underline{1}), \\
& (\underline{1}, \underline{10}, 0, \underline{4}, \underline{1}) .
\end{aligned}
$$

Of course, the model has $N=4$ supersymmetry and no chiral fermions.

Consider now the following shift vector $\delta=\left(\delta_{L}, \delta_{R}\right)$ acting on the lattice $\Gamma_{22 ; 6} \otimes \mathrm{E}_{8}$ :

$$
\boldsymbol{\delta}=\left(0^{5},-\frac{1}{2},\left(\frac{1}{2}\right)^{9}, 0,\left(\frac{1}{2}\right)^{2}, 0^{2},-\frac{1}{2}, \frac{1}{2} ; 0^{3},\left(\frac{1}{2}\right)^{6}, 0^{5}\right) .
$$

The part of this shift which acts in $\mathrm{SO}(12)_{\mathrm{R}}$ is equivalent to a Weyl rotation and preserves therefore superconformal invariance. However, it breaks $G_{L} \times\left[G_{R} \times E_{8}\right]_{R}$ to the following group:

$$
\mathrm{G}_{\mathrm{L}, \mathrm{R}}^{\prime}=\left[(\mathrm{SO}(10))^{3} \times \mathrm{SO}(2) \times(\mathrm{SO}(4))^{3}\right]_{\mathrm{L}} \times\left[(\mathrm{SO}(6))^{3} \times \mathrm{SO}(10)\right]_{\mathrm{R}} .
$$

The last $\mathrm{SO}(10)$ contains the Lorentz group $\mathrm{SO}(4)$ and the "ghost group" $\mathrm{SO}(6)$. The new covariant lattice $\Gamma_{22 ; 14}^{\prime}$ is the weight lattice of $G_{\mathrm{L}, \mathrm{R}}^{\prime}$ which is obtained as the direct sum $\Gamma_{0} \oplus \Gamma_{0}+\delta \oplus \Gamma_{0}+2 \delta \oplus \Gamma_{0}+3 \delta$.

Investigating the conjugacy classes of $\Gamma_{22 ; 14}^{\prime}$ we recognize that the constraint vector $\boldsymbol{T}^{\prime}$ of superconformal invariance is not invariant under the shift and is not any longer a lattice vector of $\Gamma_{22 ; 14}^{\prime}$. Instead, there are new invariant constraint vectors $\boldsymbol{T}^{\prime \prime}$ of the form eq. (3.8) which are elements of the $(0,0,0,0,0,0,0 ; \mathrm{V}, \mathrm{V}, \mathrm{V}, \mathrm{V})$ conjugacy class of $\Gamma_{22 ; 14}^{\prime}$. This change in constraint vectors is necessary to obtain chiral fermions. Furthermore, all 4 gravitinos are projected out, such that the resulting model is non-supersymmetric.

Finally, we are able to construct the chiral spectrum for this model. The lattice vector $\boldsymbol{p}_{1}$ of eq. (4.16) leads to chiral fermions in the unshifted sector transforming under $\left[(\operatorname{SO}(10))^{3} \times \mathrm{SO}(2) \times \mathrm{SO}(4)^{3}\right]_{\mathrm{L}} \otimes\left[(\mathrm{SO}(6)]_{\mathrm{R}}^{3}\right.$

$$
\left(\underline{1}, \underline{1}, \underline{16},-\frac{1}{2}, \underline{2}, \underline{1}, \underline{1} ; \underline{1}, \underline{1}, \underline{4}\right) \text {. }
$$

These are the only massless fermions in the unshifted sector of the theory. However, there are also chiral fermions coming from the shifted sector. The vector $\boldsymbol{p}_{2}+3 \boldsymbol{\delta}$ leads to massless fermions transforming as

$$
\left(\underline{1}, \underline{16}, \underline{1},-\frac{1}{2}, \underline{1}, \underline{2}, \underline{1} ; \underline{1}, \underline{4}, \underline{1}\right)
$$


and finally the vector $\boldsymbol{p}_{3}+3 \delta$ results in the following representation:

$$
\left(\underline{16}, \underline{1}, \underline{1},-\frac{1}{2}, \underline{1}, \underline{1}, \underline{2} ; \underline{4}, \underline{1}, \underline{1}\right) \text {. }
$$

The full fermion content is given in refs. [4,23].

The massless scalars in the unshifted sector result from $\boldsymbol{p}_{4}, \boldsymbol{p}_{5}, \boldsymbol{p}_{6}$. Again, the shifted sector leads to further massless scalars - the full spectrum of scalars can be found in ref. $[4,23]$.

The number of massless chiral fermions in $[\mathrm{SO}(10)]^{3} \times \mathrm{U}(1)$ representations has its origin in the transformation properties under $\left[D_{2}\right]_{L}^{3} \otimes\left[D_{3}\right]_{R}^{3}$ and corresponds to the degeneracy factor for the asymmetric orbifold. The vertex operators for all fields are expressed entirely in terms of free bosons. In ref. [23] various amplitudes were calculated like Yukawa couplings, scalar self-interactions etc. for this model.

Finally, it is interesting to investigate whether arbitrary choice of background parameters can reduce the rank of the (too large) gauge symmetry. For example, the fermions are only chiral with respect to $[\mathrm{SO}(10)]^{3} \times \mathrm{U}(1)$. Therefore, one might expect that continuous variation of the background parameters breaks the gauge symmetry $[\mathrm{SO}(4)]^{3}$ via the described Higgs mechanism.

\section{Summary}

In this paper we have discussed the relation between the covariant lattice approach and a specific class of orbifolds, both describing four-dimensional heterotic string theories. We showed that asymmetric orbifolds, obtained by modding out a Weyl rotation from the lattice defining the torus compactification are equivalently described by self-dual covariant lattices for a special choice of background parameters of the six-dimensional torus.

We have discussed the constraints of superconformal invariance and the mechanisms of space-time supersymmetry breaking. The equivalence of orbifolds and covariant lattices is very useful in order to explain the number of chiral fermions in terms of group theoretical properties of self-dual lattices. Furthermore, it provides an explicit construction of twist field operators which makes the calculation of scattering amplitudes easier.

Although 4-dimensional string theories are most probably described by a large class of (super) conformal field theories which are very difficult to classify in general, it is still very useful to consider a subclass of them like asymmetric orbifolds or covariant lattices. These subclasses correspond to very special choices of (background) parameters of more general conformal field theories and are characterized by a dramatic enhancement of symmetries. The reason is that these theories are essentially described by free bosons or fermions leading to large representations of (gauge, global) symmetry groups. The use of this simplified description of four dimensional string theories lies in the fact that covariant lattices 
provide an explicit framework to calculate scattering amplitudes and, therefore, the (classical) effective action of the massless modes in an almost model independent way [23]. One obtains for example the gravity part of the effective action which remains still true for much more complicated backgrounds. On the other hand, vertex operators for (chiral) fermions and massless scalars necessarily involve also the internal degrees of freedom in order to have the correct conformal dimension 1. Here, the covariant lattices allow explicit calculation of the relevant amplitudes. Thus, general conclusions about the scalar potential can be drawn. For other amplitudes the internal part reflects itself in topological or group theoretical selection rules. Information about possible space-time supersymmetry constrains the effective action even further [38].

We would like to thank W. Lerche, H.P. Nilles and P. Vecsernyés for useful discussions. One of us (D.L.) expresses his gratitude to the California Institute of Technology where part of this work was completed.

\section{References}

[1] M.B. Green, J.H. Schwarz and E. Witten, Superstring theory, vols. I and II (Cambridge University Press, 1987)

[2] K. Narain, Phys. Lett. 169B (1986) 41

[3] H. Kawai, D. Lewellen and S. Tye, Phys. Rev. Lett. 57 (1986) 1832; Nucl. Phys. B288 (1987) 1

[4] W. Lerche, D. Lüst and A.N. Schellekens, Nucl. Phys. B287 (1987) 477

[5] I. Antoniadis, C. Bachas and C. Kounnas, Nucl. Phys. B289 (1987) 87

[6] M. Mueller and E. Witten, Phys. Lett. 182B (1986) 28

[7] K.S. Narain, M.H. Sarmadi and C. Vafa, Nucl. Phys. B288 (1987) 551

[8] R. Bluhm, L. Dolan and P. Goddard, Nucl. Phys. B289 (1987) 364

[9] H. Kawai, D. Lewellen and S. Tye, Phys. Lett. 191B (1987) 63

[10] W. Lerche, B.E.W. Nilsson and A.N. Schellekens, Nucl. Phys. B294 (1987) 136

[11] L. Dixon, V. Kaplunovsky and C. Vafa, Nucl. Phys. B294 (1987) 43

[12] P. Candelas, G.T. Horowitz, A. Strominger and E. Witten, Nucl. Phys. B258 (1985) 46

[13] D.J. Gross, J. Harvey, E. Martinec and R. Rohm, Phys. Rev. Lett. 54 (1985) 502; Nucl. Phys. B256 (1985) 253; B267 (1986) 75

[14] C.G. Callan, D. Friedan, E.J. Martinec and M.J. Perry, Nucl. Phys. B262 (1985) 593

[15] K. Narain, M. Sarmadi and E. Witten, Nucl. Phys. B279 (1987) 369

[16] L. Dixon, J. Harvey, C. Vafa and E. Witten, Nucl, Phys. B261 (1985) 651; B274 (1986) 285

[17] C. Vafa, Nucl. Phys. B273 (1986) 592

[18] L.E. Ibanez, H.P. Nilles and F. Quevedo, Phys. Lett. 187B (1987) 25; Phys. Lett. 192B (1987) 332

[19] L. Ibanez, J. Mas, H.P. Nilles and F. Quevedo, preprint CERN-TH.4859/87 (1987)

[20] I. Senda and A. Sugamoto, preprint KEK87-39 (1987)

[21] W. Lerche and D. Lüst, Phys. Lett. 187B (1987) 45

[22] W. Lerche, D. Lüst and A.N. Schellekens, Phys. Lett. 181B (1986) 71

[23] D. Lüst, S. Theisen and G. Zoupanos; Nucl. Phys. B296 (1988) 800

[24] J. Balog, P. Forgács, Z. Horváth and P. Vecsernyés, Phys. Lett. 197B (1987) 395

[25] A.N. Schellekens, preprint CERN-TH.4834/87 (1987)

[26] A. Neveu and J.H. Schwarz, Nucl. Phys. B31 (1971) 86

[27] P. Ramond, Phys. Rev. D3 (1971) 2415

[28] J. Frenkel and V.G. Kac, Inv, Math. 62 (1980) 23;

P. Goddard and D. Olive, in Vertex operators in mathematics and physics, MSRI Publ. No. 3, ed. J. Lepowsky (Springer, 1984) 
[29] P. Ginsparg, Phys. Rev. D35 (1987) 648

[30] L. Dixon, D. Friedan, E. Martinec and S. Shenker, Nucl. Phys. B282 (1987) 13

[31] S. Hamidi and C. Vafa, Nucl. Phys. B279 (1987) 465

[32] J. Atick, L. Dixon and P. Griffin, preprint SLAC-PUB-4273 (1987)

[33] V. Kostelecky, O. Lechtenfeld, W. Lerche, S. Samuel and S. Watamura, Nucl. Phys. B288 (1987) 173

[34] L. Alvarez-Gaumé, P. Ginsparg, G. Moore and C. Vafa, Phys. Lett. 171B (1986) 155

[35] L.J. Dixon and J.A. Harvey, Nucl. Phys. B274 (1986) 93

[36] J. Atick, L. Dixon and A. Sen, Nucl. Phys. B292 (1987) 109

[37] A. Strominger, Phys. Rev. Lett. 55 (1985) 2547

[38] J. Lauer, D. Lüst and S. Theisen, preprint MPI-PAE/PTH 86/87 